\title{
Evgeniy Nikolaevich Savchenko and his contribution to the knowledge of Palaearctic Tipuloidea
}

\author{
VLADIMIR I. LANTSOV \\ Laboratory of Invertebrate Diversity, Institute of Ecology of Mountain Territories of Kabardino-Balkarian Scientific Centre of \\ Russian Academy of Sciences, Innesi Armand str.,37 “A”, Nalchik.360051, Russia \\ E-mail: lantsov@megalog.ru
}

\begin{abstract}
A biography of Evgeniy Savchenko, an outstanding entomologist, who made a considerable contribution to the knowledge of tipuloid dipterans of the Palaearctic. The various stages of his scientific activity are presented. He gave names to a large number of taxa of tipuloids and taniderids, 435 in all.
\end{abstract}

Key words: Evgeniy Savchenko, biography, scientific contribution, Palaearctic Tipuloidea

Thus his memory, like some holy light, Kept alive in our hearts, will improve them, For worth shall look fairer, and truth more bright, When we think how he lived but to love them. Thomas Moore (1779-1852)

The period of the second half of the XIXth and the early XXth century was marked by a rapid development of research in the natural sciences, in particular, the development of entomology. In the middle of XIX century the entomological societies of the Old and New World were founded-The Entomological Society of America, The Russian Entomological Society (both in 1859), and The Netherlands Entomological Society (founded in 1845). During these years, there was an intensive accumulation of entomological material, an activity that dates from the time of great geographical discoveries. A lot of little-studied taxa of dipteran insects, to which the Tipuloidea belong, awaited their researchers. During this period a new generation of diptera taxonomists, who studied this group, were born. In the USA - C.P. Alexander, in Russia-A.A. Stackelberg, in Holland-F.M. Van der Wulp, in Germany-B. Mannheims, in England-F.W. Edwards, in Czechia-K. Czižek, in Latvia-P. Lackschewitz, etc. Evgeniy Savchenko belongs to this group of outstanding entomologists; he made a considerably larger contribution to the knowledge of palaearctic Tipuloidea (Families Tipulidae, Limoniidae and Pediciidae).

Evgeniy Savchenko (Savtschenko) (Fig. 1) was born on July 12th, 1909 (June 29th according to the old calendar) into a working class family. (It was impossible to find out anything about his parents). After leaving school in 1929 he had to work as a handyman in construction. Since childhood, however, he was actively engaged in entomology, therefore, in 1930 he was accepted as a volunteer laboratory assistant in the Zoological museum of All-Ukrainian Academy of Sciences. In 1935 he graduated from the Biological Faculty of Kiev University (now the Taras Shevchenko National University of Kiev) without attending lectures and he got an appointment as the assistant in the All-Union Scientific-Research Institute of Sugar Beets (now the Institute for Sugar Beets). 
The Institute for Sugar Beets of the Ukrainian Academy of Agrarian Sciences was founded in 1922. It was one of the first large agricultural scientific institutions after the revolution (1917), founded for studying scientific and practical problems of breeding.

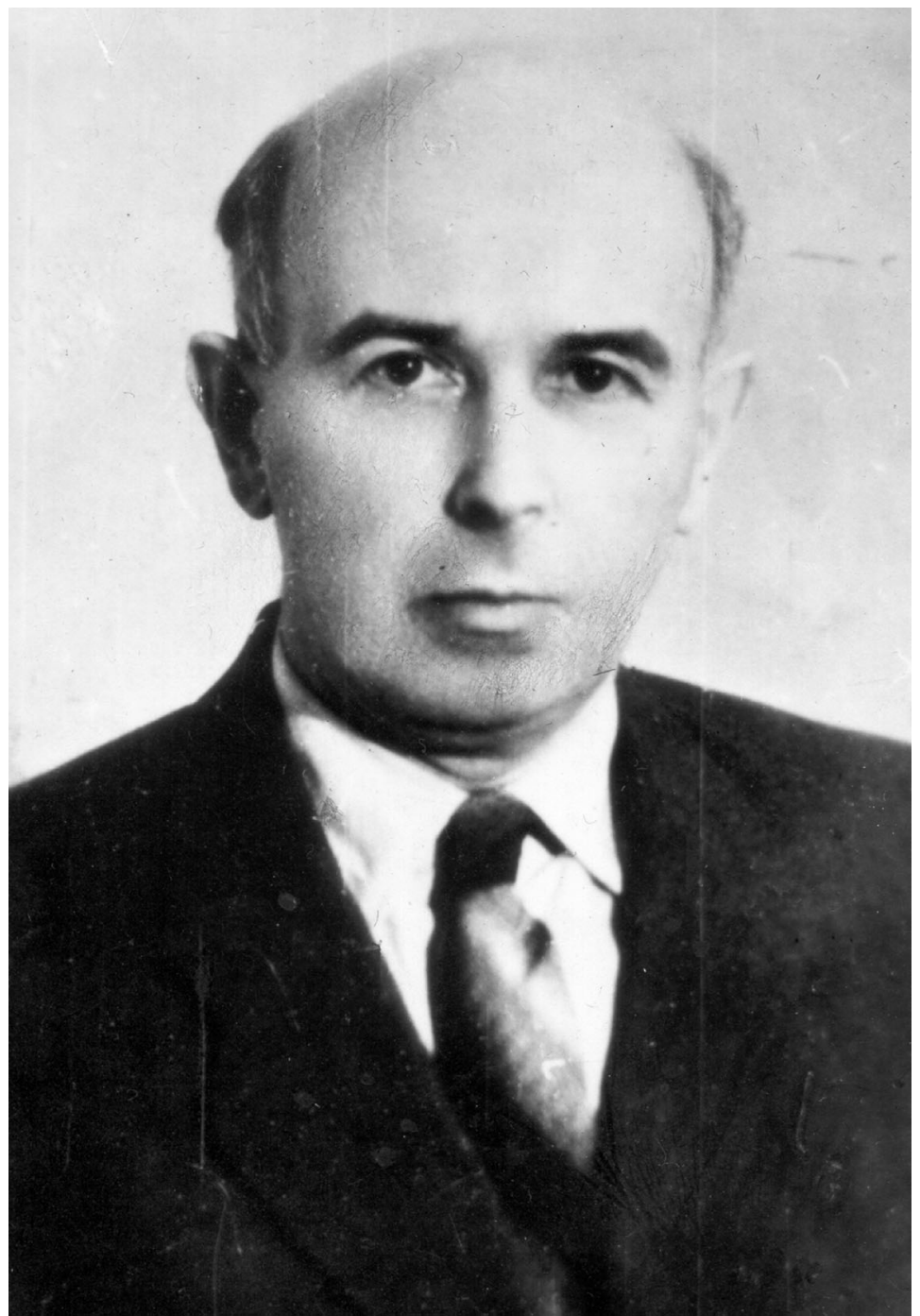

FIGURE 1. Evgeniy Nikolaevich Savchenko (1909-1994), Kiev (the picture date is not established).

Evgeniy Savchenko began his professional activity in entomology as an expert in protection of plants. In this field he published 84 works, mainly on chemical methods of controlling destructive insects including 40 publications of original research. Altogether, E. Savchenko devoted about 30 years to research in the field of applied entomology, plant protection, working in various institutions. 
His first work on entomology was published in 1930 and covered the larval stages of wireworms (Elateridae) and pseudowireworms (Tenebrionidae) populating the soils of a sugar beet cultivation area in Ukraine. In 1937 he defended his master's thesis and became the candidate of agricultural sciences in 1938. Evgeniy was engaged in working out the chemical methods of protection of plants and simultaneously studied of lamellicorn beetles (Scarabaeidae) of Ukraine.

His first work in taxonomy was on the Coleoptera, the systematics of the family Scarabaeidae. He published seven works, one of which is a monograph on the species and zoological geography of scarabeids of the Kiev Region (Savchenko 1934). The first new species that he described was in the family Scarabaeidae (Savchenko 1938). His works in the field of systematic coleopterology can be considered as the second stage of his activity as an entomologist.

Terrible years of the Second World War were approaching. As is known, Wehrmacht forces entered Kiev on September 19th, 1941. Kiev was occupied by the Nazi forces of Germany for 778 days before its liberation on November 6th, 1943. In September 1941, Savchenko could not be evacuated and remained in Kiev because, unfortunately, his wife fell badly ill. At this time he started to work at the Institute of Zoology. Then from March 1942 till October 1943 Evgeniy headed the newly founded Institute of Plant Protection in Kiev. This Institute vanished with the leaving of the Germans (personal information from L.V.Puchkova who knew E.Savchenko well since the wartime). Unlike other entomological colleagues (S.J.Paramonov, L.A.Sheljuzhko, N.S.Obraztsova and N.S.Pavlitskaja), Evgeniy did not leave Kiev at the same time as the retreating German forces in the autumn of 1943, but remained in Kiev. In 1944 the activity of the All-Union Scientific-Research Institute of Sugar Beet was resumed, and Evgeniy returned to his former work. From 1947 to 1961 he worked in the Ukranian Scientific Research Institute of the Socialist Agriculture (currently the Institute of Plant Protection of the Ukrainian Academy of Agrarian Sciences) as a senior scientific researcher and then later as the head of the department for protection of plants. Simultaneously with working out chemical means for agricultural pest control, he was also engaged in studying the crane flies of the Soviet Union.

The third stage of the scientific activity of Evgeniy Savchenko was research on tipuloid dipterans beginning with the Tipulidae and later, also the Limoniidae. The beginning of this stage can be dated 1952, when his first two articles on tipulids of Ukraine (Savchenko 1952a, 1952b), Caucasus and Iran appeared; Evgeniy was 43 at that time. Probably an incentive was the opportunity to study the collection of tipulids, made by an entomologist, Alexander Filippovich Kryshtal (1908-1985), in the Kaniv Biosphere Nature Reserve in 1950, and the considerable collections of tipulids from the Caucasus (Teberda) made in the fifties by Leo Evgeniy Arens (1890-1967). In his first two articles Savchenko gives names to nine species and describes eight of them as new.

When Savchenko began his activity as a dipterologist, there was only sketchy data on the crane flies of Ukraine and the Russian part of the Palaearctic written at the end of the XIXth century by Belke, Jaroshevckiy, Fedchenko, Gimmerthal, Sintenis, Mik, Porchinskiy, etc., and at the beginning of the XXth century to the reports of Lackschewitz (1936) on the Arctic coast of Eurasia and Stackelberg's series of works from 1922 to 1951 on the northwest of Russia (mainly the environs of St. Petersburg), and also the descriptions of several species from the Far East by C.P. Alexander (1922, 1926 etc.). Only one work had been published on the tipulid fauna of the Caucasus (Riedel 1920).

From 1952 to 1992 Savchenko explored, to a considerable degree, the Tipuloidea fauna of the former USSR and he published 200 works in all. (For a complete listing of publications see below.) This included five monographs on tipulids, six on limoniids, participation (together with Jaroslav Starý and Pjotr Oosterbroek) in compiling the first volume of the Catalogue of Palaearctic Diptera (1992), and participation in the publication of nine chapters of four collective monographs. He 
produced profound research on the Limoniidae and Pediciidae by the publication of a series of monographs on the Ukraine, Southern Primorye Territory, Southern Kuriles and Southern Sakhalin faunae and a survey monograph on these families in the territory of the former USSR.

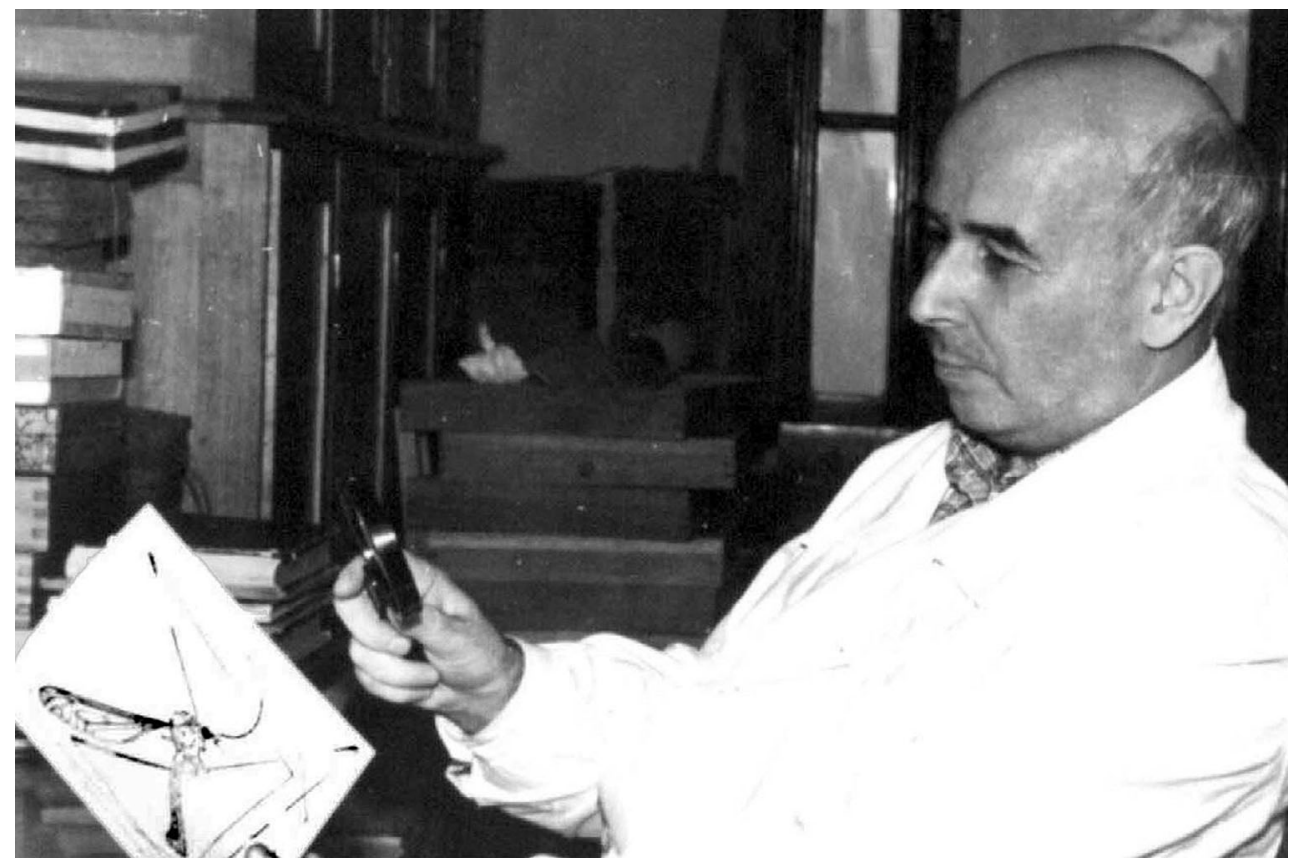

FIGURE 2. Evgeniy Nikolaevich Savchenko in the laboratory of The Institute of Zoology (Kiev, 1971).

This third period of scientific activity of E. Savchenko is divided into two overlapping stages according to years of issue of corresponding publications. The first stage comprises the research on tipulids, from 1952 to 1988 and the second stage is the research on limoniids and pediciids, from 1965 to 1992. From 1961 to 1988, Evgeniy Savchenko (Fig. 2) worked as the senior staff scientist at the Institute of Zoology, The Academy of Sciences of the Ukrainian Soviet Socialist Republic (currently the I.I. Schmalhausen Institute of Zoology of the National Academy of Sciences of Ukraine). It was the most productive period of his life, entirely devoted to studying tipuloid dipterans. Evgeniy not only received collections from his colleagues, but also participated in numerous expeditions himself to the mountains of the Caucasus (mainly in the Transcaucasus region) (Tab. 1), Central Asia and to the Far East (Primorye Territory, Sakhalin, Kuril Islands). Seventy-eight works were published concerning the Tipulidae, 115 on Limoniidae and Pediciidae, 3 on Tanyderidae and 2 on Cylindrotomidae. In his two works on the fauna of Mongolia and Bering's Island there is data on both tipulids and limoniids. Though Evgeniy's prime interest was in issues of taxonomy, in his works he paid attention to ecology, zoogeography, and phylogeny and to the description of preimaginal developmental stages. Eight works were devoted to the description of larval and pupal stages of tipulids, two works to entomopathogenic fungi, one article to the description of a new species of limoniid from Baltic amber, one to the phylogeny and evolution of Tipulidae. Evgeniy Savchenko gave names for 433 taxa of Tipuloidea and two Tanyderidae (Tab. 2; 
fig. 3, Appendix I), 410 of them were new species and subspecies. His contribution amounts to $13 \%$ of the currently known number of palaearctic species (3138) (Oosterbroek 2009). From areas of research he pointed out the majority of already known species that makes-for example for the Tipula genus-75\%. The largest number of described species is from the Caucasus (129), and the most part from the Transcaucasia (101), then Middle and Central Asia (79), and lastly the Far East and Northeast Siberia (56).

TABLE 1. The Expeditions to the regions of the former USSR of the Institute of Zoology of Ukrainian Academy of Sciences (Kiev), in which E. Savchenko took part.

\begin{tabular}{ll}
\hline \multicolumn{1}{c}{ Geographical regions } & \multicolumn{1}{c}{ Years } \\
\hline Ukraine & $1961,1962,1964,1965,1969,1970,1974,1975$ \\
$\quad$ Carpathians & 1962,1964 \\
$\quad$ Crimean Mountains & $1963,1965,1978$ \\
The West Caucasus & \\
$\quad$ Black Sea shoe & \\
Transcaucasus & 1966 \\
Georgia & 1978 \\
$\quad$ Adzharia & $1967,1969,1970$ \\
$\quad$ Abkhazia & 1966 \\
$\quad$ Other regions & $1966,1967,1970$ \\
Armenia & 1977,1978 \\
Azerbaijan & 1979,1980 \\
Central Asia &
\end{tabular}

TABLE 2. Quantity of taxa of Tipuloidea (Tipulidae, Limoniidae, Pediciidae) and Tanyderidae, described or named by Evgeniy N. Savchenko.

\begin{tabular}{lccccc}
\hline \multicolumn{1}{c}{ Family } & Genus & Subgenus & Species & Subspecies & Total \\
\hline Limoniidae & 2 & 15 & 187 & 19 & 223 \\
Pediciidae & 1 & & 26 & 3 & 30 \\
Tipulidae* & 1 & 6 & 145 & 28 & 180 \\
Tanyderidae & & & 2 & & 2 \\
Total & 4 & 21 & 360 & 50 & 435 \\
\hline
\end{tabular}

* with nomen nudum

The collection of insects gathered and processed by Evgeniy was handed over to the Zoological Museum, in the same building as the Institute of Zoology. In 1996 the Museum separated from the Institute and became an independent organization. Evgeniy Savchenko's collections are now considered to be national heritage of Ukraine. According to Vera Vladimirovna Barabanova, 
technical specialist of the Museum, in the collection gathered by Evgeniy Savchenko there are about 15000 specimens of beetles, and about 31560 specimens of tipuloid dipterans, the latter includes over 2064 species. At present work is in progress on cataloguing the type material in this collection of tipuloid dipterans.

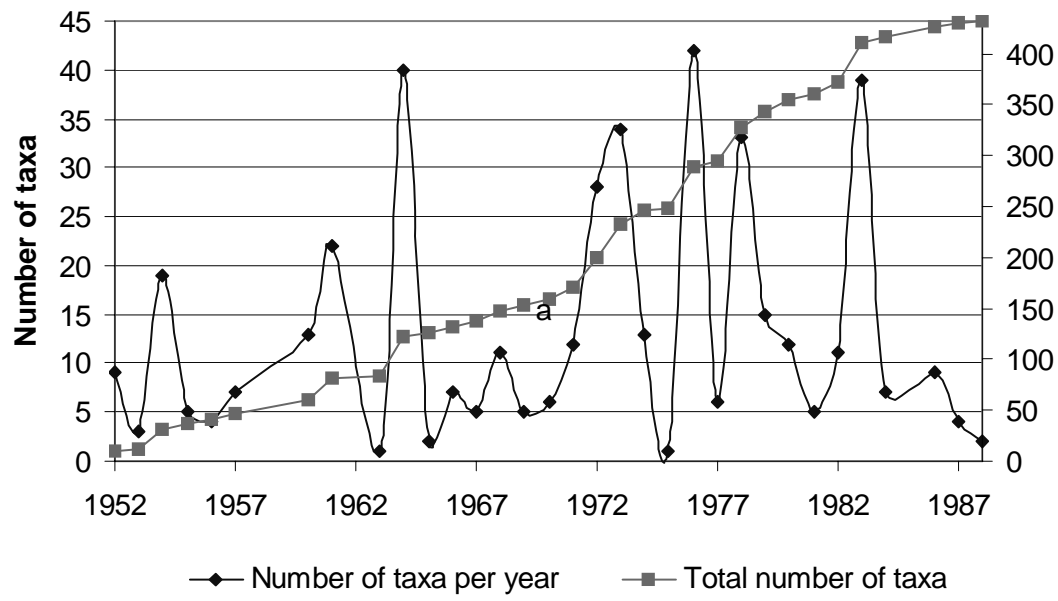

FIGURE 3. Dynamics of an increase in quantity of taxa new to science Tipulidae, Limoniidae and Pediciidae, named by E.N.Savchenko.

It is extremely important to note Evgeniy`s talent as an editor of scientific texts. He balanced exclusive attentiveness to the author's style with insistence on accuracy of facts with regard to the material. He was the editor of a considerable number of monographs in the series «Fauna of Ukraine» and the associate editor of the «Zoology Bulletin» journal. Thanks to his accurate and benevolent criticism, entomological monographic reviews were polished to perfection on a number of important in the scientific and practical relation groups.

In spite of his reputation, Evgeniy never was officially given his due; he received neither an appropriate rank nor government awards, nor substantial material encouragement. He lived very modestly in an old semibasement apartment in a shabby house constructed before the revolutionary events of 1917. Later he moved to a new apartment that was also very modest and not at all reflecting his status as a scientist with a worldwide reputation. Most likely the occupational period in his biography was the reason for that as well as the absence of comprehensive biographical data at the Institute where he worked for many years. Personal records of workers of the Institute of Zoology (as well as in any other official institute) are usually stored for a long time, but Evgeniy Nikolaevich's personal records, including his activities during the Second World War and during the post-war period, for some reason vanished completely.

The only personal record remaining dates from September $25^{\text {th }}, 1936$ and ends on January 1st, 1937. It includes only a title page and one additional page with scanty information (place and date of birth, speciality). Half of this page has numerous crossed out sections in the columns indicating that he was not a member of any political party including C.P.S.U.(B.) (Communist Party of the Soviet Union [Bolsheviks]). The rest of the 14 pages of his personal records have vanished. All Evgeniy 
Nikolaevich's requests to prepare a post-graduate student to be his successor in the study of tipuloid dipterans remained unrequited. As a result, the research of this interesting species-rich taxonomical group in Ukraine, unfortunately, did not receive the continuation.

Evgeniy Nikolaevich was married to Mokrzhitskaya Evgeniya Nikolaevna. She was the assistant at the Institute of Sugar Industry. She did not work by that time before the Second World War in 1936. Evgeniy Nikolaevna was ill and died before long at the end of seventies.

During the last seven years of his life, Evgeniy Nikolaevich did not work at the Institute any more. He had long been a widower and lived a solitary life that was brightened up by walks with an irrepressible dog, french bulldog, and by infrequent visits from his few colleagues.

The author of this article was a post-graduate student and had the honour of communicating with Evgeniy Savchenko, and personally spent some evenings with him in his old house in Ovruchskay № $21 / 23$, and then had a long correspondence with him. The last time when there was a chance to speak with Savchenko was in 1993, in his new apartment, when Fenja Brodo came to Kiev. Evgeniy welcomed us but that could not be said of his canine friend. He asked what language is more comfortable for the guest to communicate- they chose German. Savchenko knew several languages.

During the night, from 8th to 9th January, 1994, Evgeniy Nikolaevich Savchenko died of a heart attack. According to his will, his ashes were spread in the bottomland of the Irpen River-a tributary of the Dnieper, $27 \mathrm{~km}$ West of Kiev. Since his childhood, he liked to collect insects in this area.

In his work, Evgeniy Savchenko was noted for his thorough perfection of the scientific language -brevity, conciseness, and correctness, consistency of formulations, expressiveness and style. He was able to work in any situation. These qualities are inherent in true scientists, people who are devoted not simply to a science, but to searching for the truth.

All his life was devoted to studying a most surprising and remarkable phenomenon on the Earth, the variety of biota. The latter is so great that the life of one person is hardly enough to read just several pages of this fascinating book-a name to which is a biological diversity of tipuloid dipterans.

\section{Acknowledgements}

Author thank Anatoliy Kotenko and Valery Korneyev (Institute of Zoology, of the National Academy of Sciences of Ukraine, Kiev) for the photos of Evgeniy Savchenko and for important information on Savchenko's biography. My special thanks to Pjotr Oosterbroek (Zoölogisch Museum Nederland) for his constant support and help in preparing the species list and list of publications. Author express gratitude to Fenja Brodo (Ottawa, Canada) who kindly checked and improved the English text. Sincerely grateful to the workers of the Zoological Museum of the National Museum of Natural History of the National Academy of Sciences of Ukraine (Kiev), for the opportunity to be funded to work in the museum with Evgeniy Savchenko's collection. The work has been supported by the grant of Program of Presidium of the RAS "Biodiversity".

\section{Obituaries devoted to E.N. Savchenko}

Korneyev, V. A. (1995) Evgeniy Nikolaevich Savchenko (1909-1994). Journal of Ukrainian Entomological Society, 1(3-4), 3-10 (in Russian).

Lantsov, V. I. \& Byers, G. W. (1997) In memory of Prof. Evgeniy Nikolaevich Savchenko (1909-1994). International Journal of Dipterological Research, 8(2), 55-67 (in English). 
Lantsov, V. I. \& Korneyev, V. A. (2009) Evgeniy Nikolaevich Savchenko. Vestnik Zoologii, 3, $283-284$ (in Russian).

Korneyev, V. A.,. Lantsov, V. I. (2009) Evgeniy Nikolaevich Savchenko. Journal of Ukrainian Entomological Society, (in press) (in Ukrainian).

\section{References}

Alexander, C.P. (1922) The Crane-flies (Superfamily Tipuloidea, Order Diptera). In: Report of the scientific results of the Norwegian expedition to Novaya Zemlya 1921. Pt. 5. Videnskapsselskapet, Kristiania, pp. 1-16.

Alexander, C.P. (1926). Diptera collected by the late Alien Crawford on Wrangel Island. The Tipulidae. Canadian Entomologist, 58(12), 289-293.

Lackschewitz, P. (1936) Das Genus Tipula (Diptera, Nematocera) in der Arctis und dem borealen Waldgebieten Eurasien. Proceedings of Zoological Institute of Academy of Science of the USSR, 4(2), 245-312.

Oosterbroek, P. (2009) Catalogue of the Craneflies of the World (CCW). Online database, available from http:// ip30.eti.uva.nl/ccw/ (latest update: 3 September 2009).

Riedel M, (1920) Nematocera polyneura aus dem Kaukasus. Zoologische Jahrbücher, Abteilung für Systematik, $43,13-22$.

Savchenko, E.N. (1934) The fauna of leef-beetles of the Kiev region and its zoogeography. Travaux du museée zoologique, Kiev, 13, 41-85 (in Ukrainian with Russian and German summaries).

Savchenko, E.N. (1938) Description of male of Aphodius troitskii Jakobs. The Russian Entomological Review, 27(3-4), 243-245 (in Russian).

Savchenko, E.N. (1952a) A new species of crane-fly (Diptera, Tipulidae) from the Ukrainian SSR. Entomologicheskoe Obozrenie, 32, 307-310 (in Russian).

Savchenko, E.N. (1952b) New species of crane-flies (Diptera, Tipulidae) of the Caucasus and northern Iran. Trudy Zoologicheskogo Instituta Academii Nauk SSSR, Leningrad, 12, 321-349 (in Russian).

Savchenko, E.N., Oosterbroek, P. \& Starý, J. (1992) Family Limoniidae. In: Catalogue of Palaearctic Diptera, 1, Trichoceridae - Nymphomyiidae. Hungarian Natural History Museum, Budapest, pp. 183-369. 


\section{Appendix 1}

Taxa of Tanyderidae and Tipuloidea described or named by Evgeniy N. Savchenko

\section{Abbreviations}

The biogeographical regions of the species and subspecies are abbreviated as EP (East Palaearctic), NA (Nearctic), OR (Oriental), P (Palaearctic) and WP (West Palaearctic).

\section{Tanyderidae}

1. Protanyderus stackelbergi Savchenko, 1971, Entomological review. 50, 167 (as Protanyderus) (EP).

2. Protanyderus riedeli Savchenko, 1974, Zool. Zh., 53 (12), 1892 (as Protanyderus) (P).

\section{Tipuloidea}

Tipuloidea taxa described or named by Savchenko includes 433 names (Tab. 2), constituting 4 genera, 21 subgenera, 358 species and 50 subspeces. Of these, 15 are preoccupied (11 of which were replaced by Savchenko himself and four by other authors) and 21 are synonyms (including one nomen nudum, Dictenidia fuscicosta). In the list below only the 397 recognized taxa are numbered, the preoccupied names and synonyms are not numbered.

\section{Limoniidae, Chioneinae}

1 Arctoconopa australis Savchenko, 1982, In: Savchenko \& Rybin, 1982, Ent. Obozr., 61, 392 (as Arctoconopa) (EP).

2 Arctoconopa forcipata kostjukovi Savchenko, 1971, Vestn. Zool., 1971(1), 16 (as Arctoconopa) (EP).

3 Arctoconopa insulana Savchenko, 1971, Vestn. Zool., 1971(1), 14 (as Arctoconopa) (EP).

4 Baeoura mediofiligera Savchenko, 1984, In: Savchenko \& Plyushch, 1984, Ent. Obozr., 63, 174 (as Baeoura) (EP).

5 Beringomyia Savchenko, 1980, In: Savchenko \& Parkhomenko, 1980, Freshwater Fauna Far East, Vladivostok, 99.

6 Beringomyia politonigra Savchenko, 1980, In: Savchenko \& Parkhomenko, 1980, Freshwater Fauna Far East, Vladivostok, 103 (as Beringomyia) (EP).

7 Cheilotrichia (Cheilotrichia) aemula Savchenko, 1974, Dopov. Akad. Nauk Ukr. RSR (B), 1974, 943 (as Cheilotrichia) (WP).

8 Cheilotrichia (Cheilotrichia) vagans Savchenko, 1972, Dopov. Akad. Nauk Ukr. RSR (B), 1972, 560 (as Cheilotrichia) (WP).

9 Chionea (Chionea) pusilla Savchenko, 1983, Limoniidae South Primorye, 71 (as Chionea) (EP).

10 Ellipteroides (Protogonomyia) quadridens (Savchenko, 1972), Zool. Zh., 51, 1331 (as Gonomyia) (WP).

-- $\quad$ Erioconopa jaroslavi Savchenko, 1980, Ent. Obozr., 59, 395 (as Erioconopa) (EP; syn. of tadzika (Savchenko, 1972)).

11 Erioconopa tadzika (Savchenko, 1972), Zool. Zh., 51, 679 (as Erioptera) (EP).

12 Erioptera (Erioptera) biaculeata Savchenko, 1981, Zool. Zh., 60, 1585 (as Erioptera) (WP).

13 Erioptera (Erioptera) cervula Savchenko, 1972, Zool. Zh., 51, 673 (as Erioptera) (EP).

14 Erioptera (Erioptera) cornuta Savchenko, 1984, In: Savchenko \& Plyushch, 1984, Ent. Obozr., 63, 176 (as Erioptera) (EP).

-- $\quad$ Erioptera (Erioptera) cristata Savchenko, 1972, Zool. Zh., 51, 674 (as Erioptera) (EP; preoccupied by Alexander, 1956; replaced by the new name laticrista Savchenko, 1977).

15 Erioptera (Erioptera) gorgona Savchenko, 1981, Zool. Zh., 60, 1587 (as Erioptera) (WP).

16 Erioptera (Erioptera) laticrista Savchenko, 1977, Trudy Biol.-Pochv. Inst. DVNC AN SSSR, Vladivostok, (N.S.) 45(148), 100 (as Erioptera) (EP). 
Erioptera (Erioptera) mabelanoides Savchenko, 1972, Zool. Zh., 51, 671 (as Erioptera) (EP; syn. of horii Alexander, 1924).

17 Erioptera (Erioptera) phoinix Savchenko, 1972, Zool. Zh., 51, 676 (as Erioptera) (WP).

18 Erioptera (Erioptera) quadrihamata Savchenko, 1972, Zool. Zh., 51, 677 (as Erioptera) (EP).

19 Erioptera (Erioptera) tenuirama Savchenko, 1972, Zool. Zh., 51, 677 (as Erioptera) (WP).

20 Erioptera (Mesocyphona) conica (Savchenko, 1972), Vestn. Zool., 1972(5), 29 (as Mesocyphona) (P).

21 Erioptera (Mesocyphona) lilliputina (Savchenko, 1972), Vestn. Zool., 1972(5), 30 (as Mesocyphona) (EP).

22 Erioptera (Mesocyphona) spinifera (Savchenko, 1972), Vestn. Zool., 1972(5), 28 (as Mesocyphona) (EP).

23 Gnophomyia ctenura Savchenko, 1976, In: Savchenko \& Krivolutskaya, 1976, Limoniidae South Kuril Is and South Sakhalin, 104 (as Gnophomyia) (EP).

24 Gonempeda armata Savchenko, 1971, Dopov. Akad. Nauk Ukr. RSR (B), 1971, 949 (as Gonempeda) (WP).

25 Gonomyia (Gonomyia) lobulata Savchenko, 1980, Vestn. Zool., 1980(3), 37 (as Gonomyia) (EP).

26 Gonomyia (Gonomyia) modica Savchenko, 1972, Zool. Zh., 51, 1337 (as Gonomyia) (WP).

27 Gonomyia (Gonomyia) nigrotuberculata Savchenko, 1986, Vestn. Zool., 1986(1), 31 (as Gonomyia) (EP).

28 Gonomyia (Gonomyia) odontostyla Savchenko, 1972, Zool. Zh., 51, 1336 (as Gonomyia) (EP).

29 Gonomyia (Gonomyia) papposa Savchenko, 1983, Zool. Zh., 62, 643 (as Gonomyia) (WP).

30 Gonomyia (Gonomyia) serpentigera Savchenko, 1983, Limoniidae South Primorye, 101 (as Gonomyia) (EP).

31 Gonomyia (Gonomyia) sexlobata Savchenko, 1978, In: Savchenko \& Parkhomenko, 1978, Vestn. Zool., 1978(1), 32 (as Gonomyia) (EP).

32 Gonomyia (Gonomyia) spinula Savchenko, 1983, Limoniidae South Primorye, 100 (as Gonomyia) (EP).

33 Gonomyia (Gonomyia) subtenella Savchenko, 1972, Zool. Zh., 51, 1339 (as Gonomyia) (WP).

34 Gonomyia (Gonomyia) tristylata Savchenko, 1983, Limoniidae South Primorye, 100 (as Gonomyia) (EP).

35 Gonomyia (Gonomyia) vana Savchenko, 1980, Vestn. Zool., 1980(3), 36 (as Gonomyia) (EP).

36 Gonomyia (Idiocerodes) Savchenko, 1972, Zool. Zh., 51, 1330.

37 Gonomyia (Idiocerodes) diabarica Savchenko, 1972, Zool. Zh., 51, 1334 (as Gonomyia) (P).

38 Gonomyia (Leiponeura) melanorhyncha Savchenko, 1987, Zool. Zh., 66, 304 (as Gonomyia) (EP).

39 Gonomyia (Prolipophleps) Savchenko, 1972, Zool. Zh., 51, 1331.

40 Gonomyia (Prolipophleps) brachiostyla Savchenko, 1972, Zool. Zh., 51, 1340 (as Gonomyia) (WP).

-- Gonomyia (Teuchogonomyia) curvistyla Savchenko, 1982, In: Savchenko \& Rybin, 1982, Ent. Obozr., 61, 394 (as Gonomyia) (EP; preoccupied by Alexander, 1961; replaced by the new name gulchona Oosterbroek, 2009).

41 Gonomyia (Teuchogonomyia) quadrilobata Savchenko, 1983, Limoniidae South Primorye, 101 (as Gonomyia) (EP).

42 Gonomyodes similissimus Savchenko, 1980, Vestn. Zool., 1980(1), 20 (as Gonomyodes) (EP).

43 Hesperoconopa acutistyla Savchenko, 1980, Vestn. Zool., 1980(1), 22 (as Hesperoconopa) (EP).

44 Hoplolabis (Lunaria) Savchenko, 1982, Fauna Ukrainy, 14(3), 182.

45 Hoplolabis (Lunaria) idiophallus (Savchenko, 1973), Zbirn. Prats Zool. Mus., Kiev, 35, 38 (as Ilisia) (WP).

46 Hoplolabis (Parilisia) Savchenko, 1976, In: Savchenko \& Krivolutskaya, 1976, Limoniidae South Kuril Is and South Sakhalin, 91.

47 Hoplolabis (Parilisia) albibasis (Savchenko, 1983), Vestn. Zool., 1983(4), 3 (as Ilisia) (EP).

48 Hoplolabis (Parilisia) caudata (Savchenko, 1983), Vestn. Zool., 1983(4), 6 (as Ilisia) (EP). 
Hoplolabis (Parilisia) forcipula (Savchenko, 1978), Vestn. Zool., 1978(5), 45 (as Ilisia) (EP).

Hoplolabis (Parilisia) incisurata (Savchenko, 1982), Fauna Ukrainy, 14(3), 181 (as Ilisia) (WP; syn. of mannheimsi (Mendl, 1974)).

50 Hoplolabis (Parilisia) latiloba (Savchenko, 1978), Vestn. Zool., 1978(5), 45 (as Ilisia) (EP).

51 Hoplolabis (Parilisia) obtusiapex (Savchenko, 1982), Fauna Ukrainy, 14(3), 175 (as Ilisia) (WP).

52 Hoplolabis (Parilisia) obtusirama (Savchenko, 1983), Vestn. Zool., 1983(4), 7 (as Ilisia) (EP).

53 Hoplolabis (Parilisia) pontica (Savchenko, 1984), Vestn. Zool., 1984(1), 88 (as Ilisia) (WP).

54 Hoplolabis (Parilisia) serratofalcata (Savchenko, 1983), Vestn. Zool., 1983(4), 6 (as Ilisia) (EP).

55 Hoplolabis (Parilisia) variegata (Savchenko, 1983), Vestn. Zool., 1983(4), 5 (as Ilisia) (EP).

56 Idiocera (Euptilostena) multipunctata Savchenko, 1982, In: Savchenko \& Rybin, 1982, Ent. Obozr., 61, 395 (as Idiocera) (EP).

57 Idiocera (Idiocera) bidens Savchenko, 1979, Dopov. Akad. Nauk Ukr. RSR (B), 1979, 770 (772 in Russian Doklady ed.) (as Idiocera) (WP).

58 Idiocera (Idiocera) octoapiculata (Savchenko, 1972), Zool. Zh., 51, 1333 (as Gonomyia) (WP).

59 Idiocera (Idiocera) theowaldi Savchenko, 1982, In: Savchenko \& Rybin, 1982, Ent. Obozr., 61,396 (as Idiocera) (EP).

60 Ilisia parchomenkoi Savchenko, 1974, Zool. Zh., 53, 474 (as Ilisia) (P).

61 Molophilus (Molophilus) aequiramus Savchenko, 1982, Vestn. Zool., 1982(4), 81 (as Molophilus) (WP).

-- $\quad$ Molophilus (Molophilus) aequistylus Savchenko, 1979, Dopov. Akad. Nauk Ukr. RSR (B), 1979, 680 (683 in Russian Doklady ed.) (as Molophilus) (WP; preoccupied by Alexander, 1927; replaced by the new name aequiramus Savchenko, 1982).

62 Molophilus (Molophilus) atrostylus Savchenko, 1978, Ent. Obozr., 57, 413 (as Molophilus) (P).

63 Molophilus (Molophilus) bidigitifer Savchenko, 1976, Ent. Obozr., 55, 449 (as Molophilus) (P).

64 Molophilus (Molophilus) crimensis Savchenko, 1976, Ent. Obozr., 55, 448 (as Molophilus) (WP).

65 Molophilus (Molophilus) ermolenkoi Savchenko, 1976, Dopov. Akad. Nauk Ukr. RSR (B), 1976, 370 (373 in Russian Doklady ed.) (as Molophilus) (WP).

66 Molophilus (Molophilus) hyrcanus Savchenko, 1978, Ent. Obozr., 57, 403 (as Molophilus) (WP).

67 Molophilus (Molophilus) kotenkoi Savchenko, 1986, Vestn. Zool., 1986(1), 29 (as Molophilus) (WP).

68 Molophilus (Molophilus) lackschewitzianus hebetatus Savchenko, 1976, Ent. Obozr., 55, 446 (as Molophilus) (WP).

69 Molophilus (Molophilus) longistylus Savchenko, 1976, In: Savchenko \& Krivolutskaya, 1976, Limoniidae South Kuril Is and South Sakhalin, 101 (as Molophilus) (EP).

70 Molophilus (Molophilus) macracanthus Savchenko, 1983, Limoniidae South Primorye, 89 (as Molophilus) (EP).

71 Molophilus (Molophilus) multilobatus Savchenko, 1976, Ent. Obozr., 55, 440 (as Molophilus) (EP).

72 Molophilus (Molophilus) nodulifer Savchenko, 1978, Ent. Obozr., 57, 399 (as Molophilus) (WP).

73 Molophilus (Molophilus) palpifer Savchenko, 1976, Ent. Obozr., 55, 444 (as Molophilus) (WP).

74 Molophilus (Molophilus) pauper Savchenko, 1978, Ent. Obozr., 57, 409 (as Molophilus) (WP).

75 Molophilus (Molophilus) politonigrus Savchenko, 1983, Vestn. Zool., 1983(1), 52 (as Molophilus) (WP).

76 Molophilus (Molophilus) ponticus Savchenko, 1982, Fauna Ukrainy, 14(3), 212 (as Molophilus) (WP).

77 Molophilus (Molophilus) serratus Savchenko, 1976, Ent. Obozr., 55, 441 (as Molophilus) (EP).

78 Molophilus (Molophilus) stroblianus decoloratus Savchenko, 1978, Ent. Obozr., 57, 409 (as Molophilus) (WP).

79 Molophilus (Molophilus) subgriseus Savchenko, 1976, Ent. Obozr., 55, 446 (as Molophilus) (WP).

80 Molophilus (Molophilus) subochraceus Savchenko, 1976, Ent. Obozr., 55, 443 (as Molophilus) (WP).

81 Molophilus (Molophilus) urodontus brachyurus Savchenko, 1978, Ent. Obozr., 57, 406 (as Molophilus) (WP). 
Molophilus (Molophilus) urodontus cephaliger Savchenko, 1978, Ent. Obozr., 57, 407 (as Molophilus) (WP).

Molophilus (Molophilus) urodontus urodontus Savchenko, 1978, Ent. Obozr., 57, 403 (as Molophilus) (P).

84 Molophilus (Promolophilus) avazhon Savchenko, 1976, Ent. Obozr., 55, 439 (as Molophilus) (EP). Ormosia (Oreophila) longicornis Savchenko, 1980, Ent. Obozr., 59, 396 (as Ormosia) (EP).

South Kuril Is and South Sakhalin, 95 and 96 (as Ormosia) (EP).

\section{Ormosia (Ormosia) amicorum Savchenko \& Tomov, 1975, Dopov. Akad. Nauk Ukr. RSR (B), 1975,} 948 (950 in Russian Doklady ed.) (as Ormosia) (WP).

Ormosia (Ormosia) autumna Savchenko, 1976, In: Savchenko \& Krivolutskaya, 1976, Limoniidae South Kuril Is and South Sakhalin, 95 and 98 (as Ormosia) (EP).

Ormosia (Ormosia) helifera Savchenko, 1978, In: Savchenko \& Parkhomenko, 1978, Vestn. Zool., 1978(1), 30 (as Ormosia) (EP).

94 Ormosia (Ormosia) inaperta Savchenko, 1976, In: Savchenko \& Krivolutskaya, 1976, Limoniidae South Kuril Is and South Sakhalin, 95 (as Ormosia) (EP).

Ormosia (Ormosia) inflexa Savchenko, 1973, Ent. Obozr., 52, 440 (as Ormosia) (EP).

Ormosia (Ormosia) levanidovae Savchenko, 1983, Limoniidae South Primorye, 84 (as Ormosia) (EP).

Ormosia (Ormosia) longispina Savchenko, 1983, Vestn. Zool., 1983(1), 18 (as Ormosia) (WP).

Ormosia (Ormosia) lotida Savchenko, 1973, Ent. Obozr., 52, 443 (as Ormosia) (WP).

Ormosia (Ormosia) microstyla Savchenko, 1973, Ent. Obozr., 52, 451 (as Ormosia) (WP).

Ormosia (Ormosia) multidentata curvata Savchenko, 1973, Ent. Obozr., 52, 455 (as Ormosia) (EP; preoccupied by Alexander, 1924; replaced by the new name sankhobensis Oosterbroek, 2009). (EP).

Ormosia (Ormosia) orientobifida Savchenko, 1983, Limoniidae South Primorye, 85 (as Ormosia) (EP). Ormosia (Ormosia) rostrifera Savchenko, 1973, Ent. Obozr., 52, 446 (as Ormosia) (WP).

Ormosia (Ormosia) serrata Savchenko, 1973, Ent. Obozr., 52, 453 (as Ormosia) (WP).

Ormosia (Ormosia) subfascipennis Savchenko, 1973, Ent. Obozr., 52, 444 (as Ormosia) (WP).

Ormosia (Ormosia) subserrata Savchenko, 1976, Zool. Zh., 55, 304 (as Ormosia) (WP).

Phyllolabis ghilarovi Savchenko, 1983, Vestn. Zool., 1983(1), 15 (as Phyllolabis) (WP).

Phyllolabis tjederi Savchenko, 1967, Vestn. Zool., 1967(4), 17 (as Phyllolabis) (WP).

Rhabdomastix (Rhabdomastix) leucophaea Savchenko, 1976, Dopov. Akad. Nauk Ukr. RSR (B), 1976, 561 (562 in Russian Doklady ed.) (as Rhabdomastix) (WP).

Rhypholophus imitator Savchenko, 1981, Vestn. Zool., 1981(1), 24 (as Rhypholophus) (EP).

Rhypholophus intermixtus (Savchenko, 1973), Ent. Obozr., 52, 456. (as Ormosia) (WP).

Rhypholophus simulans (Savchenko, 1973), Ent. Obozr., 52, 458 (as Ormosia) (WP).

Scleroprocta acifurca Savchenko, 1979, Dopov. Akad. Nauk Ukr. RSR (B), 1979, 769 (771 in Russian Doklady ed.) (as Scleroprocta) (WP).

113 Scleroprocta latiprocta Savchenko, 1973, Ent. Obozr., 52, 461 (as Scleroprocta) (WP).

114 Symplecta (Psiloconopa) alexanderi (Savchenko, 1973), Dopov. Akad. Nauk Ukr. RSR (B), 1973, 569 (as Erioptera) (WP).

115 Symplecta (Psiloconopa) beringiana Savchenko, 1979, Dopov. Akad. Nauk Ukr. RSR (B), 1979, 1053 (1054 in Russian Doklady ed.) (as Symplecta) (EP).

116 Symplecta (Psiloconopa) trilaciniata Savchenko, 1982, Vestn. Zool., 1982(2), 70 (as Symplecta) (EP). 
117 Tasiocera (Dasymolophilus) exigua Savchenko, 1973, Zbirn. Prats Zool. Mus., Kiev, 35, 39 (as Tasiocera) (WP).

\section{Limoniidae, Dactylolabinae}

118 Dactylolabis (Bothrophorus) Savchenko, 1984, In: Savchenko \& Plyushch, 1984, Ent. Obozr., 63, 169.

119 Dactylolabis (Bothrophorus) monstrosa (Savchenko, 1971), Dokl. Akad. Nauk Tadzhik SSR, 4, 58 (as Austrolimnophila) (EP).

120 Dactylolabis (Coenolabis) Savchenko, 1969, Vestn. Zool., 1969(6), 36.

121 Dactylolabis (Coenolabis) aberrans aberrans Savchenko, 1963, Dopov. Akad. Nauk Ukr. RSR (B), 1963, 129 (as Dactylolabis) (WP).

122 Dactylolabis (Coenolabis) aberrans arsianensis Savchenko, 1969, Vestn. Zool., 1969(6), 39 (as Dactylolabis) (WP).

123 Dactylolabis (Coenolabis) aberrans imeretica Savchenko, 1969, Vestn. Zool., 1969(6), 39 (as Dactylolabis) (WP).

124 Dactylolabis (Dactylolabis) carbonaria Savchenko, 1972, Insects Mongolia, 1, 726 (as Dactylolabis) (EP).

125 Dactylolabis (Dactylolabis) cingulata Savchenko, 1978, Zool. Zh., 57, 1180 (as Dactylolabis) (WP).

126 Dactylolabis (Dactylolabis) degradans Savchenko, 1978, Zool. Zh., 57, 1185 (as Dactylolabis) (EP).

127 Dactylolabis (Dactylolabis) dilatatoides Savchenko, 1978, Zool. Zh., 57, 1181 (as Dactylolabis) (EP).

128 Dactylolabis (Dactylolabis) grunini Savchenko, 1978, Zool. Zh., 57, 1183 (as Dactylolabis) (EP).

129 Dactylolabis (Dactylolabis) hirtipes Savchenko, 1978, Zool. Zh., 57, 1184 (as Dactylolabis) (EP).

130 Dactylolabis (Dactylolabis) laticellula Savchenko, 1978, Zool. Zh., 57, 1182 (as Dactylolabis) (EP).

131 Dactylolabis (Dactylolabis) opaca Savchenko, 1978, Vestn. Zool., 1978(5), 42 (as Dactylolabis) (EP).

132 Dactylolabis (Dactylolabis) retrograda Savchenko, 1978, Zool. Zh., 57, 1175 (as Dactylolabis) (WP).

133 Dactylolabis (Dactylolabis) satanas Savchenko, 1971, Ent. Obozr., 50, 163 (as Dactylolabis) (EP).

\section{Limoniidae, Limnophilinae}

134 Adelphomyia acicularis bidens Savchenko, 1983, Limoniidae South Primorye, 53 (as Adelphomyia) (EP).

135 Afrolimnophila abludens (Savchenko, 1971), Vestn. Zool., 1971(6), 14 (as Limnophila) (WP).

136 Afrolimnophila basispina (Savchenko, 1971), Vestn. Zool., 1971(6), 13 (as Limnophila) (WP).

137 Afrolimnophila minima (Savchenko, 1971), Vestn. Zool., 1971(6), 11 (as Limnophila) (WP).

138 Austrolimnophila (Archilimnophila) arborea Savchenko, 1978, Dopov. Akad. Nauk Ukr. RSR (B), 1978, 377 (380 in Russian Doklady ed.) (as Austrolimnophila) (EP).

139 Austrolimnophila (Archilimnophila) subpolaris Savchenko, 1969, Dopov. Akad. Nauk Ukr. RSR (B), 1969, 1117 (as Austrolimnophila) (P).

-- Austrolimnophila (Austrolimnophila) ligulata Savchenko, 1976, In: Savchenko \& Krivolutskaya, 1976, Limoniidae South Kuril Is and South Sakhalin, 55 (as Austrolimnophila) (EP; syn. of asiatica (Alexander, 1925)).

140 Dicranophragma (Brachylimnophila) extremiboreale (Savchenko, 1978), Trudy Biol.-Pochv. Inst. DVNC AN SSSR, Vladivostok, (N.S.) 49(152), 64 (as Neolimnomyia) (EP).

141 Dicranophragma (Mixolimnomyia) Savchenko, 1979, Dopov. Akad. Nauk Ukr. RSR (B), 1979, 484.

142 Dicranophragma (Mixolimnomyia) rufulum (Savchenko, 1979), Dopov. Akad. Nauk Ukr. RSR (B), 1979, 482 (484 in Russian Doklady ed.) (as Neolimnomyia) (WP).

143 Eloeophila verrucosa Savchenko, 1976, In: Savchenko \& Krivolutskaya, 1976, Limoniidae South Kuril Is and South Sakhalin, 60 (as Eloeophila) (EP).

144 Hexatoma (Cladolipes) haiasana (Savchenko, 1972), Dopov. Akad. Nauk Ukr. RSR (B), 1972, 469 (as Hexatoma) (WP).

145 Hexatoma (Cladolipes) simplex asiatica (Savchenko, 1972), Dopov. Akad. Nauk Ukr. RSR (B), 1972, 
467 (as Hexatoma) (P).

146 Hexatoma (Eriocera) caesia Savchenko, 1979, Vestn. Zool., 1979(1), 25 (as Hexatoma) (EP).

-- Hexatoma (Eriocera) canescens Savchenko, 1976, In: Savchenko \& Krivolutskaya, 1976, Limoniidae

South Kuril Is and South Sakhalin, 77 and 78 (as Hexatoma) (EP; preoccupied by Alexander, 1949; replaced by the new name caesia Savchenko, 1979).

147 Hexatoma (Eriocera) superba Savchenko, 1976, In: Savchenko \& Krivolutskaya, 1976, Limoniidae South Kuril Is and South Sakhalin, 77 (as Hexatoma) (EP).

148 Hexatoma (Hexatoma) nubeculosa longivena Savchenko, 1972, Insects Mongolia, 1, 729 (as Hexatoma) (EP).

149 Paradelphomyia (Oxyrhiza) brevifurca Savchenko, 1976, Zool. Zh., 55, 389 (as Paradelphomyia) (WP).

150 Paradelphomyia (Oxyrhiza) interposita Savchenko, 1976, Zool. Zh., 55, 390 (as Paradelphomyia) (WP).

151 Paradelphomyia (Oxyrhiza) laterostriata Savchenko, 1976, Zool. Zh., 55, 387 (as Paradelphomyia) (WP).

152 Paradelphomyia (Oxyrhiza) pygmaea Savchenko, 1973, Dopov. Akad. Nauk Ukr. RSR (B), 1973, 280 (as Paradelphomyia) (WP).

153 Phylidorea (Macrolabina) Savchenko, 1986, Vestn. Zool., 1986(5), 20.

154 Phylidorea (Macrolabina) latistyla Savchenko, 1986, Vestn. Zool., 1986(5), 25 (as Phylidorea) (EP).

155 Phylidorea (Macrolabina) nigronotata macrocera Savchenko, 1986, Vestn. Zool., 1986(5), 23 (as Phylidorea) (EP).

156 Phylidorea (Paraphylidorea) Savchenko, 1986, Fauna Ukrainy, 14(2), 290.

157 Phylidorea (Phylidorea) gracilistyla Savchenko, 1973, Dopov. Akad. Nauk Ukr. RSR (B), 1973, 372 (as Phylidorea) (WP).

158 Pilaria simulans Savchenko, 1983, Limoniidae South Primorye, 61 (as Pilaria) (EP).

159 Prionolabis mendli Savchenko, 1983, Limoniidae South Primorye, 58 (as Prionolabis) (EP).

160 Prionolabis subcognata Savchenko, 1971, Dopov. Akad. Nauk Ukr. RSR (B), 1971, 754 (as Prionolabis) (WP).

161 Pseudolimnophila (Pseudolimnophila) melanura Savchenko, 1984, In: Savchenko \& Plyushch, 1984, Ent. Obozr., 63, 170 (as Pseudolimnophila) (EP).

\section{Limoniidae, Limoniinae}

162 Antocha (Antocha) biacus Savchenko, 1981, Vestn. Zool., 1981(1), 27 (as Antocha) (EP).

163 Antocha (Antocha) hirtipes Savchenko, 1971, Dopov. Akad. Nauk Ukr. RSR (B), 1971, 855 (as Antocha) (WP).

164 Antocha (Antocha) ramulifera Savchenko, 1983, Limoniidae South Primorye, 107 (as Antocha) (EP).

165 Atypophthalmus (Atypophthalmus) multisetosus Savchenko, 1983, Limoniidae South Primorye, 123 (as Atypophthalmus) (EP).

166 Atypophthalmus (Microlimonia) Savchenko, 1976, In: Savchenko \& Krivolutskaya, 1976, Limoniidae South Kuril Is and South Sakhalin, 147.

167 Dicranomyia (Dicranomyia) atropos Savchenko, 1983, Limoniidae South Primorye, 134 (as Dicranomyia) (EP).

168 Dicranomyia (Dicranomyia) clathrata Savchenko, 1984, Tax. Zoog. Insects, Kiev, 99 (as Dicranomyia) (EP).

-- Dicranomyia (Dicranomyia) clotho Savchenko, 1983, Limoniidae South Primorye, 133 (as Dicranomyia) (EP; preoccupied by Alexander, 1964; replaced by the new name kandybinae Savchenko, 1987).

169 Dicranomyia (Dicranomyia) dichroa Savchenko, 1974, Vestn. Zool., 1974(4), 65 (as Dicranomyia) (EP). 
170 Dicranomyia (Dicranomyia) distendens pallida Savchenko, 1983, Limoniidae South Primorye, 130 (as Dicranomyia) (EP).

171 Dicranomyia (Dicranomyia) kandybinae Savchenko, 1987, In: Kandybina et al., 1987, Cat. TypeSpecies Zool. Inst. Leningrad, 10 (as Dicranomyia) (EP).

172 Dicranomyia (Dicranomyia) lachesis Savchenko, 1983, Limoniidae South Primorye, 133 (as Dicranomyia) (EP).

173 Dicranomyia (Dicranomyia) limonioides Savchenko, 1974, Dopov. Akad. Nauk Ukr. RSR (B), 1974, 470 (as Dicranomyia) (EP).

174 Dicranomyia (Dicranomyia) melanantha Savchenko, 1984, Tax. Zoog. Insects, Kiev, 98 (as Dicranomyia) (WP).

175 Dicranomyia (Dicranomyia) subdichroa Savchenko, 1974, Dopov. Akad. Nauk Ukr. RSR (B), 1974, 471 (as Dicranomyia) (EP).

176 Dicranomyia (Dicranomyia) tessulata (Savchenko, 1974), Vestn. Zool., 1974(4), 68 (as Rhipidia) (EP).

177 Dicranomyia (Erostrata) Savchenko, 1976, In: Savchenko \& Krivolutskaya, 1976, Limoniidae South Kuril Is and South Sakhalin, 130.

178 Dicranomyia (Glochina) brevispina Savchenko, 1976, In: Savchenko \& Krivolutskaya, 1976, Limoniidae South Kuril Is and South Sakhalin, 141 (as Dicranomyia) (EP).

179 Dicranomyia (Glochina) kaszabi (Mannheims \& Savchenko, 1973), Annls Hist.-Nat. Mus. Natn. Hung., 65, 260 (as Limonia) (EP).

180 Dicranomyia (Glochina) persordida Savchenko, 1976, In: Savchenko \& Krivolutskaya, 1976, Limoniidae South Kuril Is and South Sakhalin, 142 (as Dicranomyia) (EP).

181 Dicranomyia (Idiopyga) Savchenko, 1987, Vestn. Zool., 1987(6), 81.

182 Dicranomyia (Idiopyga) melleicauda stenoptera Savchenko, 1970, Dopov. Akad. Nauk Ukr. RSR (B), 1970, 468 (as Dicranomyia) (WP).

183 Dicranomyia (Idiopyga) violovitshi Savchenko, 1974, Vestn. Zool., 1974(4), 67 (as Dicranomyia) (EP).

-- Dicranomyia (Salebria) Savchenko, 1976, In: Savchenko \& Krivolutskaya, 1976, Limoniidae South Kuril Is and South Sakhalin, 140 (Preoccupied by Zeller, 1856; replaced by the new name Salebriella Savchenko, 1978).

-- Dicranomyia (Salebriella) Savchenko, 1978, Vestn. Zool., 1978(5), 49 (Syn. of Dicranomyia (Glochina) Meigen, 1830).

-- Dicranomyia (Sphaeropyga) Savchenko, 1976, In: Savchenko \& Krivolutskaya, 1976, Limoniidae South Kuril Is and South Sakhalin, 146. (Preoccupied by Baier, 1960; replaced by the new name Idiopyga Savchenko, 1987).

184 Dicranoptycha mirabilis Savchenko, 1970, Dopov. Akad. Nauk Ukr. RSR (B), 1970, 564 (as Dicranoptycha) (EP).

185 Dicranoptycha recurvispina Savchenko, 1974, Vestn. Zool., 1974(6), 31 (as Dicranoptycha) (WP).

186 Elephantomyia (Elephantomyia) krivosheinae Savchenko, 1976, Dopov. Akad. Nauk Ukr. RSR (B), 1976, 467 (470 in Russian Doklady ed.) (as Elephantomyia) (P).

187 Elephantomyia (Elephantomyia) zonata Savchenko, 1976, In: Savchenko \& Krivolutskaya, 1976, Limoniidae South Kuril Is and South Sakhalin, 74 (as Elephantomyia) (EP).

188 Helius (Helius) corniger Savchenko, 1983, Limoniidae South Primorye, 65 (as Helius) (EP).

189 Helius (Helius) gibbifer Savchenko, 1981, Vestn. Zool., 1981(1), 23 (as Helius) (EP).

190 Helius (Helius) longirostris ussuriensis Savchenko, 1983, Limoniidae South Primorye, 65 (as Helius) (EP).

191 Libnotes (Libnotes) infumosa Savchenko, 1983, Limoniidae South Primorye, 112 (as Libnotes) (EP).

192 Limonia bidens Savchenko, 1979, Ent. Obozr., 58, 153 (as Limonia) (EP).

193 Limonia eos Stary \& Savchenko, 1976, Acta Ent. Bohemoslov., 73, 183 (as Limonia) (WP).

194 Limonia gissarica Savchenko, 1979, Ent. Obozr., 58, 155 (as Limonia) (EP).

195 Limonia messaurea boreoorientalis Savchenko, 1987, Vestn. Zool., 1987(6), 78 (as Limonia) (EP). 
Limonia nemoralis Savchenko, 1983, Limoniidae South Primorye, 146 (as Limonia) (EP).

Limonia nigropunctata intermixta Savchenko, 1976, In: Savchenko \& Krivolutskaya, 1976, Limoniidae South Kuril Is and South Sakhalin, 154 (as Limonia) (EP).

Limonia pullata aquila Savchenko, 1983, Limoniidae South Primorye, 145 (as Limonia) (EP).

Limonia subaequalis Savchenko, 1979, Ent. Obozr., 58, 158 (as Limonia) (WP).

Limonia zebrina Savchenko, 1979, Ent. Obozr., 58, 156 (as Limonia) (WP; syn. of splendens (Kuntze, 1920)).

Metalimnobia (Metalimnobia) dualis Savchenko, 1986, Vestn. Zool., 1986(1), 32 (as Metalimnobia) (EP).

Metalimnobia (Metalimnobia) lanceolata Savchenko, 1983, Limoniidae South Primorye, 142 (as Metalimnobia) (EP).

2 Metalimnobia (Metalimnobia) tenua Savchenko, 1976, In: Savchenko \& Krivolutskaya, 1976, Limoniidae South Kuril Is and South Sakhalin, 151 (as Metalimnobia) (P).

Orimarga (Orimarga) longiventris Savchenko, 1974, Vestn. Zool., 1974(6), 32 (as Orimarga) (WP). Rhipidia (Rhipidia) degradans Savchenko, 1983, Limoniidae South Primorye, 118 (as Rhipidia) (EP).

Rhipidia (Rhipidia) reductispina Savchenko, 1983, Limoniidae South Primorye, 117 (as Rhipidia) (EP).

Rhipidia (Rhipidia) uniseriata lutea Savchenko, 1974, Vestn. Zool., 1974(4), 72 (as Rhipidia) (EP).

Rhipidia (Rhipidia) lucea Savchenko, 1974, Vestn. Zool., 1974(4), 70 (as Rhipidia) (WP).

Thaumastoptera (Thaunastoptera) $(\mathrm{P})$.

-- $\quad$ Ulugbekia Savchenko, 1970, Dopov. Akad. Nauk Ukr. RSR (B), 1970, 564 (Syn. of Dicranoptycha Osten Sacken, 1860).

\section{Pediciidae, Pediciinae}

209 Dicranota (Dicranota) bicornigera Savchenko, 1978, Vestn. Zool., 1978(5), 40 (as Dicranota) (EP).

210 Dicranota (Dicranota) mannheimsi Savchenko, 1972, Dopov. Akad. Nauk Ukr. RSR (B), 1972, 182 (as Dicranota) (EP).

211 Dicranota (Dicranota) quadrihamata Savchenko, 1977, Trudy Biol.-Pochv. Inst. DVNC AN SSSR, Vladivostok, (N.S.) 46(149), 78 (as Dicranota) (EP).

212 Dicranota (Dicranota) retrorsa Savchenko, 1972, Insects Mongolia, 1, 723 (as Dicranota) (EP).

213 Dicranota (Eudicranota) sibirica immaculata Savchenko, 1989, Limoniidae USSR, 21 (as Dicranota) (EP).

214 Dicranota (Eudicranota) sibirica pallidicosta Savchenko, 1976, In: Savchenko \& Krivolutskaya, 1976, Limoniidae South Kuril Is and South Sakhalin, 36 (as Dicranota) (EP).

215 Dicranota (Ludicia) aberrans Savchenko, 1980, In: Savchenko \& Parkhomenko, 1980, Freshwater Fauna Far East, Vladivostok, 96 (as Dicranota) (EP).

216 Dicranota (Paradicranota) concavista Savchenko, 1977, Trudy Biol.-Pochv. Inst. DVNC AN SSSR, Vladivostok, (N.S.) 46(149), [Ispravleniya] Erratum (as Dicranota) (EP).

-- Dicranota (Paradicranota) emarginata Savchenko, 1977, Trudy Biol.-Pochv. Inst. DVNC AN SSSR, Vladivostok, (N.S.) 46(149), 80 (as Dicranota) (EP; preoccupied by Alexander, 1945; replaced by the new name concavista Savchenko, 1977).

217 Dicranota (Paradicranota) hirtitergata Savchenko, 1979, Zool. Zh., 58, 1422 (as Dicranota) (WP).

218 Dicranota (Paradicranota) parviuncinata Savchenko, 1983, In: Savchenko \& Parkhomenko, 1983, Zool. Zh., 62, 1353 (as Dicranota) (WP).

-- Dicranota (Paradicranota) securifera Savchenko, 1986, Fauna Ukrainy, 14(2), 186 (as Dicranota) (WP; syn. of martinovskyi Stary, 1974).

219 Dicranota (Paradicranota) spiralis Savchenko, 1980, Ent. Obozr., 59, 392 (as Dicranota) (EP).

220 Dicranota (Rhaphidolabis) commutata Savchenko, 1976, In: Savchenko \& Krivolutskaya, 1976, 
Limoniidae South Kuril Is and South Sakhalin, 44 (as Dicranota) (EP).

221 Dicranota (Rhaphidolabis) complicata Savchenko, 1979, Vestn. Zool., 1979(1), 24 (as Dicranota) (EP).

222 Dicranota (Rhaphidolabis) ferruginea Savchenko, 1983, Vestn. Zool., 1983(1), 13 (as Dicranota) (EP).

223 Dicranota (Rhaphidolabis) mesasiatica Savchenko, 1973, Dopov. Akad. Nauk Ukr. RSR (B), 1973, 568 (as Dicranota) (EP).

224 Dicranota (Rhaphidolabis) squarrosa Savchenko, 1976, In: Savchenko \& Krivolutskaya, 1976, Limoniidae South Kuril Is and South Sakhalin, 43 (as Dicranota) (EP).

-- $\quad$ Eugenia Savchenko, 1977, Dopov. Akad. Nauk Ukr. RSR (B), 1977, 566 (Preoccupied by Gould, 1856; replaced by the new name Savchenkoiana Kocak, 1981).

225 Nipponomyia gracilis Savchenko, 1983, Limoniidae South Primorye, 34 (as Nipponomyia) (EP).

226 Pedicia (Amalopis) pallida Savchenko, 1976, In: Savchenko \& Krivolutskaya, 1976, Limoniidae South Kuril Is and South Sakhalin, 30 (as Pedicia) (EP).

-- Pedicia (Amalopis) reductiloba Savchenko, 1976, In: Savchenko \& Krivolutskaya, 1976, Limoniidae South Kuril Is and South Sakhalin, 29 (as Pedicia) (EP; syn. of depressiloba Alexander, 1945).

227 Pedicia (Amalopis) tenuiloba grisea Savchenko, 1976, In: Savchenko \& Krivolutskaya, 1976, Limoniidae South Kuril Is and South Sakhalin, 27 and 30 (as Pedicia) (EP).

228 Pedicia (Crunobia) dispar Savchenko, 1978, Zool. Zh., 57, 700 (as Pedicia) (WP).

-- $\quad$ Pedicia (Crunobia) elegantula Savchenko, 1978, Zool. Zh., 57, 703 (as Pedicia) (WP; syn. of persica Alexander, 1975).

229 Pedicia (Crunobia) lobifera Savchenko, 1986, Fauna Ukrainy, 14(2), 159 (as Pedicia) (WP).

230 Pedicia (Crunobia) pallens Savchenko, 1978, Zool. Zh., 57, 703 (as Pedicia) (WP).

231 Pedicia (Crunobia) semireducta Savchenko, 1978, Zool. Zh., 57, 706 (as Pedicia) (WP).

232 Pedicia (Crunobia) staryi Savchenko, 1978, Zool. Zh., 57, 709 (as Pedicia) (WP).

233 Savchenkoiana mokrzhitskae (Savchenko, 1977), Dopov. Akad. Nauk Ukr. RSR (B), 1977, 566 (568 in Russian Doklady ed.) (as Eugenia) (EP).

\section{Tipulidae, Ctenophorinae}

234 Ctenophora (Xiphuromorpha) Savchenko, 1973, Fauna USSR, Dipt., 2(5), (N.S.) 105, 248.

-- Dictenidia fuscicosta Savchenko, 1966, In: Savchenko \& Krivolutskaya, 1966, Entomofauna Lesov, Moscow, 1966, 57 (as Dictenidia) (EP; nomen nudum; syn. of pictipennis fumicosta Savchenko, 1973).

235 Dictenidia pictipennis fumicosta Savchenko, 1973, Fauna USSR, Dipt., 2(5), (N.S.) 105, 197 (as Dictenidia) (EP).

236 Tanyptera (Mesodictenidia) stackelbergiana Savchenko, 1973, Fauna USSR, Dipt., 2(5), (N.S.) 105, 260 (as Tanyptera) (EP).

-- $\quad$ Tanyptera (Protanyptera) Savchenko, 1973, Fauna USSR, Dipt., 2(5), (N.S.) 105, 257 (as ) (Syn. of Tanyptera (Mesodictenidia) Matsumura, 1931).

237 Tanyptera (Tanyptera) atrata przewalskii Savchenko, 1973, Fauna USSR, Dipt., 2(5), (N.S.) 105, 272 (as Tanyptera) (EP).

\section{Tipulidae, Dolichopezinae}

238 Dolichopeza (Dolichopeza) hirsuticauda Savchenko, 1968, Ent. Obozr., 47, 914 (as Dolichopeza) (WP).

239 Dolichopeza (Dolichopeza) katoi rufula Savchenko, 1979, Land Arthropods Far East, 108 (as Dolichopeza) (EP).

240 Dolichopeza (Nesopeza) imitator Savchenko, 1979, Land Arthropods Far East, 114 (as Dolichopeza) (EP).

241 Dolichopeza (Oropeza) barbigera (Savchenko, 1980), Tax. Insects Far East, 109 (as Oropeza) (EP).

242 Dolichopeza (Oropeza) modesta (Savchenko, 1980), Tax. Insects Far East, 110 (as Oropeza) (P). 


\section{Tipulidae, Tipulinae}

243 Angarotipula Savchenko, 1961, Fauna USSR, Dipt., 2(3), (N.S.) 79, 347.

244 Angarotipula rubzovi (Savchenko, 1961), Fauna USSR, Dipt., 2(3), (N.S.) 79, 358 (as Tipula) (EP).

-- Nephrotoma autumnalis (Savchenko, 1973), Fauna USSR, Dipt., 2(5), (N.S.) 105, 106 (as Pales) (EP; syn. of palloris (Coquillett, 1898)).

245 Nephrotoma barbigera (Savchenko, 1964), Pratsi Inst. Zool. Akad. Nauk Ukr. RSR, Kiev 20, 186 (as Pales) (EP).

246 Nephrotoma biappendiculata (Savchenko, 1973), Fauna USSR, Dipt., 2(5), (N.S.) 105, 167 (as Pales) (EP).

247 Nephrotoma eugeniae (Savchenko, 1957), Ent. Obozr., 36, 216 (as Pales) (WP).

248 Nephrotoma impigra fulvovittata (Savchenko, 1964), Ent. Obozr., 43, 892 (as Pales) (OR).

249 Nephrotoma laticrista Savchenko, 1966, Bonn. Zool. Beitr., 17, 262 (as Nephrotoma) (EP).

-- Nephrotoma mannheimsi Savchenko, 1966, Bonn. Zool. Beitr., 17, 260 (as Nephrotoma) (EP; syn. of pullata (Alexander, 1914)).

-- $\quad$ Nephrotoma nigrina Savchenko, 1973, Zbirn. Prats Zool. Mus., Kiev, 35, 36 (as Nephrotoma) (WP; syn. of cornicina cornicina (Linnaeus, 1758)).

250 Nephrotoma pilicauda (Savchenko, 1973), Fauna USSR, Dipt., 2(5), (N.S.) 105, 169 (as Pales) (EP).

251 Nephrotoma quadrifaria farsidica (Savchenko, 1957), Ent. Obozr., 36, 218 (as Pales) (WP).

252 Nephrotoma relicta (Savchenko, 1973), Fauna USSR, Dipt., 2(5), (N.S.) 105, 118 (as Pales) (P).

253 Nephrotoma rubriventris (Savchenko, 1957), Ent. Obozr., 36, 219 (as Pales) (EP).

254 Nephrotoma stackelbergi (Savchenko, 1957), Ent. Obozr., 36, 211 (as Pales) (EP).

255 Nephrotoma sublunulicornis (Savchenko, 1957), Ent. Obozr., 36, 213 (as Pales) (EP).

256 Nephrotoma villosa (Savchenko, 1973), Fauna USSR, Dipt., 2(5), (N.S.) 105, 164 (as Pales) (EP).

257 Nephrotoma violovitshi (Savchenko, 1967), In: Savchenko \& Violovich, 1967, Annls Zool. Warsz., 25, 355 (as Pales) (EP).

258 Prionocera mannheimsi Savchenko, 1983, Fauna USSR, Dipt., 2(1-2), (N.S.) 127, 498 (as Prionocera) (P).

259 Prionocera subturcica Savchenko, 1983, Fauna USSR, Dipt., 2(1-2), (N.S.) 127, 490 (as Prionocera) (WP).

260 Tipula (Acutipula) macra Savchenko, 1961, Fauna USSR, Dipt., 2(3), (N.S.) 79, 414 (as Tipula) (WP).

261 Tipula (Acutipula) nigroantennata Savchenko, 1961, Fauna USSR, Dipt., 2(3), (N.S.) 79, 400 (as Tipula) (WP).

262 Tipula (Acutipula) transcaucasica Savchenko, 1961, Fauna USSR, Dipt., 2(3), (N.S.) 79, 413 (as Tipula) (P).

263 Tipula (Arctotipula) besselsi centrasiatica Savchenko, 1961, Fauna USSR, Dipt., 2(3), (N.S.) 79, 336 (as Tipula) (EP).

264 Tipula (Arctotipula) caliginosa Savchenko, 1961, Fauna USSR, Dipt., 2(3), (N.S.) 79, 339 (as Tipula) (EP).

265 Tipula (Arctotipula) crassispina Savchenko, 1978, Dopov. Akad. Nauk Ukr. RSR (B), 1978, 652 (656 in Russian Doklady ed.) (as Tipula) (EP).

266 Tipula (Arctotipula) excelsa Savchenko, 1961, Fauna USSR, Dipt., 2(3), (N.S.) 79, 323 (as Tipula) (EP).

267 Tipula (Arctotipula) hirtitergata altaica Savchenko, 1972, In: Savchenko et al., 1972, Ent. Obozr., 51, 86 (as Tipula) (EP).

268 Tipula (Arctotipula) pudibunda Savchenko, 1961, Fauna USSR, Dipt., 2(3), (N.S.) 79, 329 (as Tipula) (EP).

269 Tipula (Arctotipula) quadriloba Savchenko, 1967, In: Savchenko \& Violovich, 1967, Annls Zool. Warsz., 25, 345, 363 (as Tipula) (EP).

270 Tipula (Arctotipula) rubicunda Savchenko, 1961, Fauna USSR, Dipt., 2(3), (N.S.) 79, 331 (as Tipula) 
(EP).

271 Tipula (Beringotipula) Savchenko, 1961, Fauna USSR, Dipt., 2(3), (N.S.) 79, 471.

272 Tipula (Dendrotipula) Savchenko, 1964, Fauna USSR, Dipt., 2(4), (N.S.) 89, 458.

273 Tipula (Formotipula) melanomera gracilispina Savchenko, 1960, Ent. Obozr., 39, 888 (as Tipula) (OR).

-- $\quad$ Tipula (Geotipula) Savchenko, 1964, Fauna USSR, Dipt., 2(4), (N.S.) 89, 15 (Syn. of Tipula (Pterelachisus) Rondani, 1842).

274 Tipula (Lindnerina) bistilata rectangularis Savchenko, 1966, Entomofauna Lesov, Moscow, 1966, 93 (as Tipula) (EP).

275 Tipula (Lunatipula) adusta adusta Savchenko, 1954, Trudy Zool. Inst., Leningrad, 15, 182 (as Tipula) (P).

276 Tipula (Lunatipula) adusta lucistriata Mannheims \& Savchenko, 1967, Reichenbachia, 9, 152 (as Tipula) (P).

277 Tipula (Lunatipula) adzharolivida Savchenko, 1968, Ent. Obozr., 47, 917 (as Tipula) (WP).

-- $\quad$ Tipula (Lunatipula) alticola Savchenko, 1964, Fauna USSR, Dipt., 2(4), (N.S.) 89, 345 (as Tipula) (WP; preoccupied by Alexander, 1915; replaced by the new name altivolans Savchenko, 1971).

278 Tipula (Lunatipula) arnoldii Savchenko, 1957, Zool. Zh., 36, 1496 (as Tipula) (WP).

279 Tipula (Lunatipula) astigma Savchenko, 1968, Ent. Obozr., 47, 932 (as Tipula) (WP).

280 Tipula (Lunatipula) bactridica Savchenko, 1954, Dokl. Akad. Nauk Tadzhik SSR, 12, 51 (as Tipula) (EP).

281 Tipula (Lunatipula) borysthenica Savchenko, 1954, Zbirn. Prats Zool. Mus., Kiev, 26, 90 (as Tipula) (WP).

282 Tipula (Lunatipula) chelifera Savchenko, 1964, Fauna USSR, Dipt., 2(4), (N.S.) 89, 361 (as Tipula) (WP).

283 Tipula (Lunatipula) chloris Savchenko, 1972, In: Savchenko \& Sagdiyeva, 1972, Dopov. Akad. Nauk Ukr. RSR (B), 1972, 84 (as Tipula) (EP).

284 Tipula (Lunatipula) ctenura Savchenko, 1952, Trudy Zool. Inst., Leningrad, 12, 321 (as Tipula) (WP).

285 Tipula (Lunatipula) curvispina Savchenko, 1954, Trudy Zool. Inst., Leningrad, 15, 190 (as Tipula) (EP).

286 Tipula (Lunatipula) deserticola Savchenko, 1968, Ent. Obozr., 47, 919 (as Tipula) (WP).

287 Tipula (Lunatipula) dumetorum Savchenko, 1964, Fauna USSR, Dipt., 2(4), (N.S.) 89, 388 (as Tipula) (WP).

288 Tipula (Lunatipula) erectispina Savchenko, 1954, Trudy Zool. Mus. KDU, 4, 121 (as Tipula) (EP).

289 Tipula (Lunatipula) flabellifera Savchenko, 1954, Trudy Zool. Inst., Leningrad, 15, 188 (as Tipula) (EP).

290 Tipula (Lunatipula) geja Savchenko, 1968, Ent. Obozr., 47, 929 (as Tipula) (WP).

291 Tipula (Lunatipula) hybrida altivolans Savchenko, 1971, Zbirn. Prats Zool. Mus., Kiev, 34, 57 (as Tipula) (WP).

292 Tipula (Lunatipula) hybrida hybrida Savchenko, 1952, Trudy Zool. Inst., Leningrad, 12, 339 (as Tipula) (WP).

293 Tipula (Lunatipula) hyrcana Savchenko, 1973, Zbirn. Prats Zool. Mus., Kiev, 35, 32 (as Tipula) (WP).

294 Tipula (Lunatipula) korovini Savchenko, 1970, Dopov. Akad. Nauk Ukr. RSR (B), 1970, 371 (as Tipula) (EP).

295 Tipula (Lunatipula) latistyla Savchenko, 1954, Trudy Zool. Mus. KDU, 4, 130 (as Tipula) (WP).

296 Tipula (Lunatipula) lehriana Savchenko, 1964, Fauna USSR, Dipt., 2(4), (N.S.) 89, 280 (as Tipula) (EP).

297 Tipula (Lunatipula) lithophila Savchenko, 1968, Ent. Obozr., 47, 921 (as Tipula) (WP).

298 Tipula (Lunatipula) livida morosa Savchenko, 1964, Fauna USSR, Dipt., 2(4), (N.S.) 89, 298 (as Tipula) (WP). 
Tipula (Lunatipula) macropyga Savchenko, 1952, Trudy Zool. Inst., Leningrad, 12, 331 (as Tipula) (WP).

300 Tipula (Lunatipula) maija Savchenko, 1973, Zbirn. Prats Zool. Mus., Kiev, 35, 31 (as Tipula) (WP).

301 Tipula (Lunatipula) melanothrix Savchenko \& Theischinger, 1978, Bull. zool. Mus. Univ. Amsterdam, 6, 119 (as Tipula) (EP).

302 Tipula (Lunatipula) mima Savchenko, 1964, Fauna USSR, Dipt., 2(4), (N.S.) 89, 370 (as Tipula) (WP).

303 Tipula (Lunatipula) nocturna Savchenko, 1964, Fauna USSR, Dipt., 2(4), (N.S.) 89, 387 (as Tipula) (WP).

304 Tipula (Lunatipula) pallidicornis Savchenko, 1954, Trudy Zool. Mus. KDU, 4, 126 (as Tipula) (EP).

305 Tipula (Lunatipula) pallidithorax Savchenko, 1954, Trudy Zool. Inst., Leningrad, 15, 191 (as Tipula) (EP).

306 Tipula (Lunatipula) pararecticornis Savchenko \& Theischinger, 1978, Bull. zool. Mus. Univ. Amsterdam, 6, 120 (as Tipula) (EP).

307 Tipula (Lunatipula) parasimurg Savchenko, 1968, Ent. Obozr., 47, 927 (as Tipula) (WP).

308 Tipula (Lunatipula) quadridentata paupera Savchenko, 1964, Fauna USSR, Dipt., 2(4), (N.S.) 89, 393 (as Tipula) (WP).

309 Tipula (Lunatipula) quadridentata quadridentata Savchenko, 1964, Fauna USSR, Dipt., 2(4), (N.S.) 89, 392 (as Tipula) (WP).

310 Tipula (Lunatipula) rutila Savchenko, 1952, Trudy Zool. Inst., Leningrad, 12, 344 (as Tipula) (WP).

311 Tipula (Lunatipula) saltatrix Savchenko, 1964, Dopov. Akad. Nauk Ukr. RSR (B), 1964, 1394 (as Tipula) (WP).

312 Tipula (Lunatipula) simurg Dzhafarov \& Savchenko, 1964, In: Savchenko, 1964, Fauna USSR, Dipt., 2(4), (N.S.) 89, 370 (as Tipula) (WP).

313 Tipula (Lunatipula) strigosa Savchenko, 1952, Trudy Zool. Inst., Leningrad, 12, 342 (as Tipula) (WP).

314 Tipula (Lunatipula) subaurita Savchenko, 1964, Fauna USSR, Dipt., 2(4), (N.S.) 89, 386 (as Tipula) (WP).

315 Tipula (Lunatipula) subbispina Savchenko, 1952, Trudy Zool. Inst., Leningrad, 12, 325 (as Tipula) (WP).

316 Tipula (Lunatipula) sublunata Savchenko, 1952, Trudy Zool. Inst., Leningrad, 12, 335 (as Tipula) (WP).

317 Tipula (Lunatipula) submanca Savchenko, 1964, Fauna USSR, Dipt., 2(4), (N.S.) 89, 286 (as Tipula) (EP).

318 Tipula (Lunatipula) subrecticornis argyrea Savchenko \& Theischinger, 1978, Bull. zool. Mus. Univ. Amsterdam, 6, 119 (as Tipula) (EP).

319 Tipula (Lunatipula) subrecticornis subrecticornis Savchenko, 1964, Fauna USSR, Dipt., 2(4), (N.S.) 89, 328 (as Tipula) (EP).

320 Tipula (Lunatipula) subvelox Savchenko, 1968, Ent. Obozr., 47, 925 (as Tipula) (WP).

321 Tipula (Lunatipula) sushkini Savchenko, 1964, Fauna USSR, Dipt., 2(4), (N.S.) 89, 299 (as Tipula) (EP).

322 Tipula (Lunatipula) talyshensis Savchenko, 1964, Fauna USSR, Dipt., 2(4), (N.S.) 89, 391 (as Tipula) (WP).

323 Tipula (Lunatipula) theowaldi Savchenko, 1964, Fauna USSR, Dipt., 2(4), (N.S.) 89, 372 (as Tipula) (WP).

324 Tipula (Lunatipula) transcaspica Savchenko, 1954, Trudy Zool. Mus. KDU, 4, 119 (as Tipula) (EP).

325 Tipula (Lunatipula) valerii Savchenko, 1968, Vestn. Zool., 1968(5), 29 (as Tipula) (WP).

-- $\quad$ Tipula (Lunatipula) velox Savchenko, 1954, Zbirn. Prats Zool. Mus., Kiev, 26, 87 (as Tipula) (WP; syn. of soosi soosi Mannheims, 1954).

326 Tipula (Lunatipula) zaitzevi Savchenko, 1952, Trudy Zool. Inst., Leningrad, 12, 321 (as Tipula) (WP).

327 Tipula (Lunatipula) zarnigor Savchenko, 1954, Dokl. Akad. Nauk Tadzhik SSR, 12, 47 (as Tipula) 
(EP).

328 Tipula (Lunatipula) zimini opacithorax Savchenko, 1964, Fauna USSR, Dipt., 2(4), (N.S.) 89, 274 (as Tipula) (EP).

329 Tipula (Lunatipula) zimini semiopaca Savchenko, 1964, Fauna USSR, Dipt., 2(4), (N.S.) 89, 274 (as Tipula) (P).

330 Tipula (Lunatipula) zimini zimini Savchenko, 1954, Trudy Zool. Inst., Leningrad, 15, 185 (as Tipula) (EP).

331 Tipula (Odonatisca) Savchenko, 1956, Zbirn. Zool. Mus. KDU, 5, 130.

332 Tipula (Odonatisca) timptonensis Savchenko, 1956, Zbirn. Zool. Mus. KDU, 5, 144 (as Tipula) (EP).

-- $\quad$ Tipula (Platytipula) minuta Savchenko, 1961, Fauna USSR, Dipt., 2(3), (N.S.) 79, 84 (as Tipula) (EP; preoccupied by Muller, 1776; replaced by the new name haplostyla Oosterbroek \& Theowald, 1992).

333 Tipula (Platytipula) violovitshiana Savchenko, 1961, Fauna USSR, Dipt., 2(3), (N.S.) 79, 77 (as Tipula) (EP).

334 Tipula (Pterelachisus) carinifrons violovitshi Savchenko, 1956, Trudy Dal'nevost. Fil. Akad. Nauk SSR, Ser. Zool., 3, 177 (as Tipula) (EP).

335 Tipula (Pterelachisus) hirsutipes spinifera Savchenko, 1964, Fauna USSR, Dipt., 2(4), (N.S.) 89, 107 (as Tipula) (EP).

336 Tipula (Pterelachisus) hispida Savchenko, 1964, Fauna USSR, Dipt., 2(4), (N.S.) 89, 82 (as Tipula) (EP).

337 Tipula (Pterelachisus) interposita Savchenko, 1966, Dopov. Akad. Nauk Ukr. RSR (B), 1966, 1092 (as Tipula) (EP).

338 Tipula (Pterelachisus) kurilensis Savchenko, 1970, Ent. Obozr., 49, 116 (as Tipula) (EP).

-- $\quad$ Tipula (Pterelachisus) littoralis Savchenko, 1964, Fauna USSR, Dipt., 2(4), (N.S.) 89, 66 (as Tipula) (EP; preoccupied by Linnaeus, 1758; replaced by the new name ochotana Savchenko, 1970).

339 Tipula (Pterelachisus) luteobasalis Savchenko, 1964, Fauna USSR, Dipt., 2(4), (N.S.) 89, 76 (as Tipula) (WP).

340 Tipula (Pterelachisus) macrostyla Savchenko, 1964, Fauna USSR, Dipt., 2(4), (N.S.) 89, 64 (as Tipula) (EP).

341 Tipula (Pterelachisus) middendorffi unicolor Savchenko, 1964, Fauna USSR, Dipt., 2(4), (N.S.) 89, 41 (as Tipula) (EP).

342 Tipula (Pterelachisus) nudicellula Savchenko, 1966, In: Savchenko \& Krivolutskaya, 1966, Entomofauna Lesov, Moscow, 1966, 54 (as Tipula) (EP).

343 Tipula (Pterelachisus) ochotana Savchenko, 1970, Ent. Obozr., 49, 118 (as Tipula) (EP).

344 Tipula (Pterelachisus) octomaculata Savchenko, 1964, Fauna USSR, Dipt., 2(4), (N.S.) 89, 44 (as Tipula) (WP).

345 Tipula (Pterelachisus) pontica Savchenko, 1964, Fauna USSR, Dipt., 2(4), (N.S.) 89, 52 (as Tipula) (WP).

-- $\quad$ Tipula (Pterelachisus) scythica Savchenko, 1952, Ent. Obozr., 32, 307 (as Tipula) (WP; syn. of jutlandica Nielsen, 1947).

346 Tipula (Pterelachisus) stenostyla Savchenko, 1964, Fauna USSR, Dipt., 2(4), (N.S.) 89, 37 (as Tipula) (P).

347 Tipula (Pterelachisus) trichopleura Savchenko, 1964, Fauna USSR, Dipt., 2(4), (N.S.) 89, 68 (as Tipula) (WP).

348 Tipula (Pterelachisus) tshernovskii Savchenko, 1954, Trudy Zool. Inst., Leningrad, 15, 192 (as Tipula) (EP).

349 Tipula (Pterelachisus) tundrensis cinereoabdominalis Savchenko, 1964, Fauna USSR, Dipt., 2(4), (N.S.) 89, 104 (as Tipula) (EP).

350 Tipula (Pterelachisus) vermiculata Savchenko, 1964, Fauna USSR, Dipt., 2(4), (N.S.) 89, 81 (as Tipula) (EP). 
351 Tipula (Savtshenkia) macaronesica Savchenko, 1961, Fauna USSR, Dipt., 2(3), (N.S.) 79, 175 (as Tipula) (WP).

352 Tipula (Savtshenkia) minuscula Savchenko, 1971, Zbirn. Prats Zool. Mus., Kiev, 34, 57 (as Tipula) (EP).

-- $\quad$ Tipula (Savtshenkia) nana Savchenko, 1968, Zool. Zh., 47, 1567 (as Tipula) (EP; preoccupied by Macquart, 1834; replaced by the new name minuscula Savchenko, 1971).

353 Tipula (Savtshenkia) nivalis Savchenko, 1961, Fauna USSR, Dipt., 2(3), (N.S.) 79, 208 (as Tipula) (WP).

354 Tipula (Savtshenkia) odontostyla Savchenko, 1961, Fauna USSR, Dipt., 2(3), (N.S.) 79, 172 (as Tipula) (WP).

355 Tipula (Savtshenkia) simulans Savchenko, 1966, Fauna Ukrainy, 14(1), 199 (as Tipula) (WP).

356 Tipula (Savtshenkia) subalpium Savchenko, 1961, Fauna USSR, Dipt., 2(3), (N.S.) 79, 172 (as Tipula) (WP).

357 Tipula (Schummelia) ahrensi Savchenko, 1957, Zool. Zh., 36, 1494 (as Tipula) (WP).

358 Tipula (Schummelia) variicornis incisicauda Savchenko, 1961, Fauna USSR, Dipt., 2(3), (N.S.) 79, 106 (as Tipula) (EP).

359 Tipula (Sinotipula) chimaera Savchenko, 1969, Dopov. Akad. Nauk Ukr. RSR (B), 1969, 653 (as Tipula) (EP).

360 Tipula (Sinotipula) emiliae Savchenko, 1964, Pratsi Inst. Zool. Akad. Nauk Ukr. RSR, Kiev, 20, 182 (as Tipula) (EP).

361 Tipula (Sinotipula) schizorhyncha Savchenko, 1966, In: Savchenko \& Krivolutskaya, 1966, Entomofauna Lesov, Moscow, 1966, 51 (as Tipula) (EP).

362 Tipula (Vestiplex) aestiva Savchenko, 1960, Horae Soc. Ent. Ross., 47, 198 (as Tipula) (EP).

363 Tipula (Vestiplex) ambigua Savchenko, 1964, Fauna USSR, Dipt., 2(4), (N.S.) 89, 226 (as Tipula) (EP).

364 Tipula (Vestiplex) aptera Savchenko, 1955, Ent. Obozr., 34, 292 (as Tipula) (EP).

365 Tipula (Vestiplex) bicalcarata Savchenko, 1965, Dopov. Akad. Nauk Ukr. RSR (B), 1965, 1523 (as Tipula) (EP).

-- $\quad$ Tipula (Vestiplex) cinerascens Savchenko, 1954, Trudy Zool. Inst., Leningrad, 15, 197 (as Tipula) (EP; syn. of laccata Lundström \& Frey, 1916).

366 Tipula (Vestiplex) coronifera Savchenko, 1960, Horae Soc. Ent. Ross., 47, 210 (as Tipula) (EP).

-- $\quad$ Tipula (Vestiplex) dulkeiti Savchenko, 1954, Trudy Zool. Inst., Leningrad, 15, 195 (as Tipula) (EP; syn. of laccata Lundström \& Frey, 1916).

367 Tipula (Vestiplex) hirticeps Savchenko, 1960, Horae Soc. Ent. Ross., 47, 176 (as Tipula) (EP).

368 Tipula (Vestiplex) kiritshenkoi Savchenko, 1960, Horae Soc. Ent. Ross., 47, 213 (as Tipula) (EP).

369 Tipula (Vestiplex) kozlovi Savchenko, 1960, Horae Soc. Ent. Ross., 47, 178 (as Tipula) (EP).

370 Tipula (Vestiplex) nubila Savchenko, 1960, Horae Soc. Ent. Ross., 47, 184 (as Tipula) (EP).

371 Tipula (Vestiplex) opilionimorpha aligera Savchenko, 1956, Ent. Obozr., 35, 127 (as Tipula) (OR).

372 Tipula (Vestiplex) opilionimorpha opilionimorpha Savchenko, 1955, Ent. Obozr., 34, 294 (as Tipula) (EP).

373 Tipula (Vestiplex) pallidicosta pullata Savchenko, 1960, Horae Soc. Ent. Ross., 47, 150 (as Tipula) (WP).

374 Tipula (Vestiplex) pauxilla Savchenko, 1960, Horae Soc. Ent. Ross., 47, 157 (as Tipula) (EP).

375 Tipula (Vestiplex) semivittata dissimilis Savchenko, 1964, Fauna USSR, Dipt., 2(4), (N.S.) 89, 163 (as Tipula) (WP).

376 Tipula (Vestiplex) semivittata semivittata Savchenko, 1960, Horae Soc. Ent. Ross., 47, 148 (as Tipula) (WP).

377 Tipula (Vestiplex) setigera Savchenko, 1960, Horae Soc. Ent. Ross., 47, 161 (as Tipula) (EP).

378 Tipula (Vestiplex) tchukchi obtusidens Savchenko, 1964, Fauna USSR, Dipt., 2(4), (N.S.) 89, 205 (as Tipula) (EP). 
379 Tipula (Vestiplex) tumididens Savchenko, 1988, Vestn. Zool., 1988(3), 19 (as Tipula) (EP).

380 Tipula (Vestiplex) virgatula montivaga Savchenko, 1960, Horae Soc. Ent. Ross., 47, 194 (as Tipula) (EP).

381 Tipula (Yamatotipula) albifrons Savchenko, 1967, In: Savchenko \& Violovich, 1967, Annls Zool. Warsz., 25, 343, 362 (as Tipula) (EP).

382 Tipula (Yamatotipula) amblyostyla Savchenko, 1968, Dopov. Akad. Nauk Ukr. RSR (B), 1968, 766 (as Tipula) (EP).

383 Tipula (Yamatotipula) anceps Savchenko, 1965, New and little known species Siberian Fauna, 29 (as Tipula) (NA, EP).

384 Tipula (Yamatotipula) aviceniana Savchenko, 1954, Trudy Zool. Mus. KDU, 4, 114 (as Tipula) (EP).

385 Tipula (Yamatotipula) caucasimontana Savchenko, 1955, Zool. Zh., 34, 834 (as Tipula) (WP).

386 Tipula (Yamatotipula) freyana abscondita Savchenko, 1964, Fauna USSR, Dipt., 2(4), (N.S.) 89, 495 (as Tipula) (EP).

387 Tipula (Yamatotipula) hamata Savchenko, 1953, Trudy Zool. Mus. KDU, 3, 104 (as Tipula) (EP).

-- $\quad$ Tipula (Yamatotipula) hamatula Savchenko, 1971, Zbirn. Prats Zool. Mus., Kiev, 34, 57 (as Tipula) (EP; syn. of hamata Savchenko, 1953).

388 Tipula (Yamatotipula) incana Savchenko, 1955, Zool. Zh., 34, 832 (as Tipula) (EP).

389 Tipula (Yamatotipula) jucunda Savchenko, 1961, Fauna USSR, Dipt., 2(3), (N.S.) 79, 244 (as Tipula) (EP).

390 Tipula (Yamatotipula) lucifera Savchenko, 1954, Trudy Zool. Mus. KDU, 4, 109 (as Tipula) (P).

-- $\quad$ Tipula (Yamatotipula) monochroa Savchenko, 1961, Fauna USSR, Dipt., 2(3), (N.S.) 79, 252 (as Tipula) (EP; preoccupied by Wiedemann, 1828; replaced by the new name abscondita Savchenko, 1964).

391 Tipula (Yamatotipula) quadrivittata cinifera Savchenko, 1961, Fauna USSR, Dipt., 2(3), (N.S.) 79, 296 (1961, Zool. Zh., 40, 1895) (as Tipula) (EP).

392 Tipula (Yamatotipula) recticauda Savchenko, 1953, Trudy Zool. Mus. KDU, 3, 107 (as Tipula) (EP).

393 Tipula (Yamatotipula) shevtshenkoi Savchenko, 1954, Trudy Zool. Mus. KDU, 4, 117 (as Tipula) (EP).

394 Tipula (Yamatotipula) solitaria Savchenko, 1953, Trudy Zool. Mus. KDU, 3, 101 (as Tipula) (EP).

395 Tipula (Yamatotipula) subincana Savchenko, 1961, Fauna USSR, Dipt., 2(3), (N.S.) 79, 258 (as Tipula) (EP).

396 Tipula (Yamatotipula) subprotrusa Savchenko, 1955, Zool. Zh., 34, 830 (as Tipula) (EP).

397 Tipula (Unplaced) bispathifera Savchenko, 1960, Ent. Obozr., 39, 889 (as Tipula) (OR). 


\section{Appendix 2.}

\section{Bibliography}

This bibliography deals with Savchenko's papers on entomology only, other papers, such as on plant protection, were excluded.

A separation has been made between papers on Coleoptera, Tanyderidae and Tipuloidea (Crane flies). Furthermore, this bibliography includes all additions and corrections known to us with respect to a previously published bibliography (Lantsov \& Byers 1996).

\section{Coleoptera (Elateridae, Tenebrionidae, Scarabaeidae)}

11930 Savchenko, E.N. (1930) Materials to the knowledge of snapping beetles (Elateridae) and darkling beetles (Tenebrionidae et Alleculidae), inhabiting soils of plants of Umansky beetraising region. Magazine of the Sugar union, 11(19), 57-74 (in Russian).

21931 Savchenko, E.N. (1931) Beitrag zur Kenntnis der Scarabaeiden fauna Wolyniens. Mémoires de la Classe des Sciences Naturelles et Techniques, 5, 43-62 (in German).

31933 Savchenko, E.N. (1933) An overview of the leef-beetles (Coleoptera, Scarabaeidae) from Podilya. Travaux du museée zoologique, Kiev, 12, 121-143 (in Ukrainian with German summary).

41934 Savchenko, E.N. (1934a) The fauna of leef-beetles of the Kiev region and its zoogeography. Travaux du museée zoologique, Kiev, 13, 41-85 (in Ukrainian with Russian and German summary).

51934 Savchenko, E.N. (1934b) Notes on the leef-beetles of the Ukraine. Travaux du museée zoologique, Kiev, 13, 87-96 (in Ukrainian with Russian and German summary).

61938 Savchenko, E.N. (1938a) Description of male of Aphodius troitskii Jakobs. The Russian Entomological Review, 27 (3-4), 243-245. (in Russian).

71938 Savchenko, E.N. (1938b) Materials of the Ukr. SSR fauna Lamellicorn beetles (Coleoptera, Scarabaeidae). Academie of Sciences of Ukraine SSR, Kiev, 208 pp. (in Ukrainian).

\section{Tanyderidae}

11971 Savchenko E.N. (1971) On the discovery of Tanyderid-flies (Diptera, Tanyderidae) in Mongolia. Entomological Review, 50(1), 167-170 (in Russian).

21974 Savchenko E.N. (1974) The second species of the primitive Taniderid-flies (Diptera, Tanyderidae) from the Soviet Middle Asia. Zoologicheskiy Zhurnal, 53(12), 1892-1894 (in Russian).

31979 Savchenko E.N. (1974) Tanyderid-flies (Diptera, Tanyderidae) of the USSR fauna. Dopovidi Akademii Nauk Ukrayinskoyi RSR (B), 1979(1), 74-77 (in Ukrainian and Russian).

\section{Tipuloidea (Tipulidae, Limoniidae, Pediciidae, Cylindrotomidae)}

11952 Savchenko, E.N. (1952a) A new species of crane-fly (Diptera, Tipulidae) from the Ukrainian SSR. Entomologicheskoe Obozrenie, 32, 307-310 (in Russian).

2 Savchenko, E.N. (1952b) New species of crane-flies (Diptera, Tipulidae) of the Caucasus and northern Iran. Trudy Zoologicheskogo Instituta, Akademiya Nauk SSSR, Leningrad, 12, 321349 (in Russian).

31953 Savchenko, E.N. (1953) Three new species of crane-flies (Diptera, Tipulidae) from the Soviet Middle Asia. Trudy Zoologichnogo Museyu, Kiivskiy Derzhavniy Universitet, 3, 101-109 (in Russian).

41954 Savchenko, E.N. (1954a) New species of crane-flies (Diptera, Tipulidae) from the noneuropean parts of the USSR. Trudy Zoologicheskogo Instituta, Akademiya Nauk SSSR, Leningrad, 15, 182-198 (in Russian).

Savchenko, E.N. (1954b) On distinguishing characters of larvae of the most widespread 
species of crane-flies (Diptera, Tipulidae). Zoologicheskiy Zhurnal, 33(3), 616-636 (in Russian).

Savchenko, E.N. (1954c) Two new species of crane-flies (Diptera, Tipulidae) from the steppe region of the European part of the USSR. Zbirnyk Prats Zoolohichnoho Muzeyu, Kiev, 26, 87-94 (in Ukrainian).

Savchenko, E.N. (1954d) New species of crane-flies (Diptera, Tipulidae) from the Tadzhik SSR. Doklady Akademii Nauk Tadzhikskoy SSR, 12, 47-51 (in Russian). USSR. Trudy Zoomuzeya Kievskiy Gosudarctvennyy Universitet, 4, 109-132 (in Russian).

1955 Savchenko, E.N. (1955a) Two new species of crane-flies (Diptera, Tipulidae) with shortwinged males. Entomologicheskoe Obozrenie, 34, 287-297 (in Russian).

Savchenko, E.N. (1955b) A review of the palaearctic crane-fly (Diptera, Tipulidae) species of the Tipula aino group Alex. Zoologicheskiy Zhurnal, 34, 822-836 (in Russian).

Savchenko, E.N. (1956a) A survey of the palaearctic species of crane-flies (Diptera, Tipulidae) of the Tipula juncea Meig. group. Zbirnik Zoomuseyu, Kijiivskiy Derzhavniy Universitet im T.G.Shevchenka, 5 [Naukovi Zapiski KDU, 15(3)], 129-148 (in Ukrainian with Russian summary).

Savchenko, E.N. (1956b) On the fully winged form of Tipula (Vestiplex) opilionimorpha Sav. (Diptera, Tipulidae). Entomologicheskoe Obozrenie, 35(1), 124-129 (in Russian).

Savchenko, E.N. (1956c) Contribution to the crane-fly fauna (Diptera, Tipulidae) of the Kuril Islands. Trudy Dalnevostochnogo Filiala Imeni V.L. Komarova, Akademiya Nauk SSSR, Ser. Zooogicheskaya., 3(6), 173-180 (in Russian).

1957 Savchenko, E.N. (1957a) New palaearctic Pales Mg. (Diptera, Tipulidae). Entomologicheskoe Obozrenie, 36(1), 211-223 (in Russian and English).

Savchenko, E.N. (1957b) Two new species of crane-flies (Diptera, Tipulidae) from the North Caucasus. Zoologicheskiy Zhurnal, 36(10), 1493-1499 (in Russian).

1960 Savchenko, E.N. (1960a) A contribution to the taxonomy of crane-flies (Diptera, Tipulidae) of the subgenus Vestiplex Bezzi of the genus Tipula L. Horae Societatis Entomologicae Union Soveticae, 47, 143-216 (in Russian).

Savchenko, E.N. (1960b) Scientific results of the Chinese-Soviet zoological-botanical expeditions of 1955-1957 to South-Western China. On the crane-flies (Diptera, Tipulidae) of SW China. Entomologicheskoe Obozrenie, 39(4), 885-892 (in Russian).

1961 Savchenko, E.N. (1961a) Crane-flies (Fam. Tipulidae), Subfam. Tipulinae, Genus Tipula L., (part 1). Fauna USSR, New Series N 79, Nasekomye dvukrylye [Diptera], 2(3), 487 pp. Mosc.- Len., Izdatelstvo "Nauka" [Publishing house of Academie of Sciences of the USSR]. (in Russian).

Savchenko, E.N. (1961b) On the crane-flies fauna (Diptera, Tipulidae) of the Tuva region. Zoologicheskiy Zhurnal, 40(12), 1893-1896 (in Russian).

1962 Zinovjev, G.A. \& Savchenko, E.N. (1962) On the fauna of crane-flies (Diptera, Tipulidae) of the Amur and Zea rivers interarea and their landscape distribution. Entomologicheskoe Obozrenie, 41(3), 554-571 (in Russian).

1963 Savchenko, E.N. (1963a) New species of Dactylolabis O.-S. (Diptera, Limoniidae) from the Crimea. Dopovidi Akademii Nauk Ukrayinskoyi RSR (B), 1963(1), 129-132 (in Ukrainian). Savchenko, E.N. (1963b) On the occurrence and biology of the palaearctic crane-fly Tipula (Savtshenkia) benesignata Mnnhs. Dopovidi Akademii Nauk Ukrayinskoyi RSR (B), 1963(9), 1258-1261 (in Ukrainian with Russian and English summaries).

1964 Savchenko, E.N. (1964a) Crane-flies (Fam. Tipulidae), Subfam. Tipulinae, Genus Tipula L., (part 2). Fauna USSR, New Series N 89, Nasekomye dvukrylye [Diptera], 2(4), 503 pp. Mosc.- Len., Izdatelstvo "Nauka" [Publishing house of Academie of Sciences of the USSR]. 
(in Russian).

Savchenko, E.N. (1964b) Crane-flies of genus Tipula L. (Diptera, Tipulidae) in fauna of USSR and adjacent countries. Author's abstract dissertation of the biological sciences doctoral thesis, Kiev, $30 \mathrm{pp}$.

Savchenko, E.N. (1964c) New species of Tipula L. from the livida-group (Diptera, Tipulidae). Dopovidi Akademii Nauk Ukrayinskoyi RSR (B), 1964 (10), 1394-1397 (in Ukrainian). Savchenko, E.N. (1964d) On the taxonomy of Pales impigra (Al.) and P. pleuromaculata (Al.) (Diptera, Tipulidae). Entomologicheskoe Obozrenie, 43(4), 890-893 (in Russian). Savchenko, E.N. (1964e) Crane-flies (Diptera, Tipulidae) new for the fauna of the far-eastern USSR. Pratsi Instytutu Zoologii, Kiev, 20, 180-192 (in Ukrainian).

Savchenko, E.N. (1964f) Crane-flies (Diptera, Tipulidae) in the high mountains fauna of the Great Caucasus in Georgia. In: Fauna of the high mountains of the Great Caucasus in the Georgia region. Tbilisi, pp. 87-90 (in Russian).

1965 Chernov, Y.I. \& Savchenko, E.N. (1965) Ecology and pre-imaginal developmental phases of the arctic Tipula (Pterelachisus) carinifrons Holm. (Diptera, Tipulidae). Zoologicheskiy Zhurnal, 44(5), 777-779 (in Russian with English summary).

Savchenko, E.N. (1965a) The preimaginal phases of the crane-fly Tipula (Savtshenkia) odontostyla Sav. (Diptera, Tipulidae). Dopovidi Akademii Nauk Ukrayinskoyi RSR (B), 1965(1), 124-127 (in Ukrainian with Russian and English summaries).

Savchenko, E.N. (1965b) On the taxonomy and geographical distribution of Limonia ladogensis Lack. (Diptera, Limoniidae). Dopovidi Akademii Nauk Ukrayinskoyi RSR (B), 1965(3), 382-385 (in Ukrainian with Russian and English summaries).

Savchenko, E.N. (1965c) Limoniidae of the genus Limonia Mg., new for the fauna of the European part of the USSR. Dopovidi Akademii Nauk Ukrayinskoyi RSR (B), 1965(9), 12401243 (in Ukrainian with Russian and English summaries).

Savchenko, E.N. (1965d) A new species of crane-fly (Diptera, Tipulidae) from Magadan. In: New and little-known species of Siberian fauna. Redaktsionno-izdatelskiy otdel Sibirskogo otdeleniy AN SSSR, Novosibirsk, pp. 29-32 (in Russian).

Savchenko, E.N. (1965e) A new species of crane-fly (Diptera, Tipulidae) from the himalayensis-group. Dopovidi Akademii Nauk Ukrayinskoyi RSR (B), 1965(11), 1523-1525 (in Ukrainian with Russian and English summaries).

Savchenko, E.N. \& Koval, E.Z. (1965) Entomophilous fungi on crane-flies and limoniid-flies new for the Ukraine. Dopovidi Akademii Nauk Ukrayinskoyi RSR (B), 1965(12), 1645-1647 (in Ukrainian with Russian and English summaries).

1966 Luppova, E.P. \& Savchenko, E.N. (1966) The crane-flies (Diptera, Tipulidae) of Tadjikistan. In: The Insect Fauna and Zoogeography of Middle Asia. Izdatelstvo "Donish”, Dushanbe, pp. 178-223 (in Russian).

Savchenko, E.N. (1966a) Contribution to the crane-fly fauna (Diptera, Tipulidae) of the Ukrainian Carpathians. In: Komahi Ukrainskih Karpat i Zakarpattja. Respublik mizhividom. zbirn. Ser. "Problemy Zoologii”, Akademiya Nauk Ukrayinskoyi RSR, "Naukova Dumka", Kiev, pp. 104-122 (in Ukrainian).

Savchenko, E.N. (1966b) On the distribution, ecology and preimaginal phases of the crane fly Tipula (Mediotipula) bidens Bergr. (Diptera, Tipulidae). Entomologicheskoe Obozrenie, 45(2), 286-293 (in Russian with English summary).

Savchenko, E.N. (1966c) New Far Eastern species of Tipula L. (Diptera, Tipulidae) from the irrorata-group. Dopovidi Akademii Nauk Ukrayinskoyi RSR (B), 1966(8), 1092-1094 (in Ukrainian with Russian and English summaries).

Savchenko, E.N. (1966d) Komari-dovgonogi [Tipulidae]. Fauna Ukrajiny, Akademiy Nauk Ukrainskoy SSR, Institute of Zoology, "Naukova Dumka", Kiev 14(1), 551 pp (in 
Ukrainian).

Savchenko, E.N. (1966e) Ecology and preimaginal stages of Tipula (Savtshenkia) goriziensis Str. (Diptera, Tipulidae). Dopovidi Akademii Nauk Ukrayinskoyi RSR (B), 1966(10), 1358 1361 (in Ukrainian with Russian and English summaries).

Savchenko, E.N. (1966f) Two new eastpalaearctic species of Nephrotoma Meig. (Diptera, Tipulidae). Bonner Zoologische Beiträge, 17(3/4), 260-264.

Savchenko, E.N. (1966g) Contribution to the crane-fly fauna of the southern Magadan oblast. Entomofauna Lesov Kuril'skich Ostrovov, Kamchatki i Magadanskoi Oblasti, Izdatelstvo "Nauka", Moskva - Leningrad, 1966, pp. 90-96 (in Russian).

Savchenko, E.N. \& Krivolutskaya, G.O. (1966) On the crane-flies (Diptera, Tipulidae) of the Kuril Islands. Entomofauna Lesov Kuril'skich Ostrovov, Kamchatki i Magadanskoi Oblasti, Izdatelstvo "Nauka", Moskva - Leningrad, 1966, pp. 45-58 (in Russian).

Mannheims, B. \& Savchenko, E.N. (1967) Ergebnisse der zoologischen Forschungen von Dr.

Z. Kaszab in der Mongolei (Diptera), 105. Tipulidae. Reichenbachia, 9(17), 147-156.

Savchenko, E.N. (1967a) A new fossil limoniid-fly (Diptera, Limoniidae) from the Baltic Amber. Dopovidi Akademii Nauk Ukrayinskoyi RSR (B), 1967(5), 469-473 (in Ukrainian with Russian and English summaries).

Savchenko, E.N. (1967b) The immature stages and the taxonomic position of the crane-fly Tipula (? Subgen.) decipiens Cz. (Diptera, Tipulidae). Vestnik Zoologii, 1967(3), 22-28 (in Russian with English summary).

Savchenko, E.N. (1967c) Phyllolabis O.-S., a genus of Limoniid-flies new for the USSR (Diptera, Limoniidae). Vestnik Zoologii, 1967(4), 16-21 (in Russian).

Savchenko, E.N. \& Koval, E.Z. (1967) Interesting hypalic fungi on the crane-flies from the Ukrainian SSR. Dopovidi Akademii Nauk Ukrayinskoyi RSR (B), 1967 (9), 842-844 (in Ukrainian).

Savchenko, E.N. \& Violovich, N.A. (1967) Review of the crane-flies (Diptera, Tipulidae) of the Tuva. Annales Zoologici, Warszawa, 25(4), 317-365 (in Russian).

Savchenko, E.N. (1968a) Crane-flies (Diptera, Tipulidae) new for the fauna of the USSR. Dopovidi Akademii Nauk Ukrayinskoyi RSR (B), 1968(5), 469-472 (in Ukrainian with Russian and English summaries).

Savchenko, E.N. (1968b) A new species of the crane-fly (Diptera, Tipulidae) from the Tipula (Yamatotipula) caesia Schumm. group. Dopovidi Akademii Nauk Ukrayinskoyi RSR (B), 1968(8), 766-768 (in Ukrainian with Russian and English summaries).

Savchenko, E.N. (1968c) A new species of crane-fly (Diptera, Tipulidae) from the Georgien SSR. Vestnik Zoologii, 1968(5), 29-32 (in Russian).

Savchenko, E.N. (1968d) On a new species of crane-fly (Diptera, Tipulidae) from Kamchatka. Zoologicheskiy Zhurnal, 47(10), 1567-1570 (in Russian).

Savchenko, E.N. (1968e) New or little known species of crane-flies (Diptera, Tipulidae) from the Transcaucasus. Entomologicheskoe Obozrenie, 47(4), 912-936 (in Russian) (English translation: Entomological Review, 47(4), 557-571 (1968)).

Savchenko, E.N. \& Zapolska, T.I. (1968) New extralimital locations of the boreoalpine species of crane-flies (Diptera-Tipulidae) on the east European lowland. Dopovidi Akademii Nauk Ukrayinskoyi RSR (B), 1968 (6), 574-576 (in Ukrainian with Russian and English summaries).

Vtorov, P.P. \& Savchenko, E.N. (1968) The immature stages of three species of subgenus Vestiplex Bezzi (Diptera, Tipulidae) from the highlands of Tien Shan. Vestnik Zoologii, 1968(1), 45-63. (in Russian with English summaries).

581969 Savchenko, E.N. (1969a) New Sinotipula Al. (Diptera, Tipulidae) from the highlands of the Central Asia. Dopovidi Akademii Nauk Ukrayinskoyi RSR (B), 1969(7), 653-655 (in 
Ukrainian with Russian and English summaries).

Savchenko, E.N. (1969b) The taxonomic status, geographical distribution and intraspecific structure of the Limoniid-fly Dactylolabis aberrans Sav. (Diptera, Limoniidae). Vestnik Zoologii, 1969(6), 36-45 (in Russian).

Savchenko, E.N. (1969c) On some little-known crane-flies (Diptera, Tipulidae) of the Transcaucasus. Zbirnyk Prats Zoolohichnoho Muzeyu, Kiev, 33, 51-60 (in Ukrainian).

Savchenko, E.N. (1969d) A new subarctic species of the Limoniid flies from the genus Austrolimnophila Al. (Diptera, Limoniidae). Dopovidi Akademii Nauk Ukrayinskoyi RSR (B), 1969(2), 1117-1120 (in Ukrainian with Russian and English summaries).

Savchenko, E.N. (1969e) Fam. Cylindrotomidae. Cylindrotomid flies. In: Bei-Beinko, G.Y. (ed.), Determinative tabels of insects from the European part of the USSR, 5, Dipterans and fleas. Nauka, Leningrad, pp. 58-59 (in Russian). (See for English translation: Savchenko, 1989a.)

Savchenko, E.N. (1969f) Fam. Tipulidae. Crane-flies. In: Bei-Beinko, G.Y. (ed.), Determinative tabels of insects from the European part of the USSR, 5, Dipterans and fleas. Nauka, Leningrad, pp. 59-86 (in Russian). (See for English translation: Savchenko, 1989b.) Savchenko, E.N. (1969g) Fam. Limoniidae (Limnobiidae). Limoniid flies. In: Bei-Beinko, G.Y. (ed.), Determinative tabels of insects from the European part of the USSR, 5, Dipterans and fleas. Nauka, Leningrad, pp. 86-112 (in Russian). (See for English translation: Savchenko, 1989c.)

1970 Savchenko, E.N. (1970a) Additions on the fauna of crane-flies (Diptera, Tipulidae) from the southern Kuriles. Entomologicheskoe Obozrenie, 49(1), 108-123 (in Russian) (English translation: Entomological Review, 49(1), 61-70 (1970)).

Savchenko, E.N. (1970b) Crane-flies from the Tipula (Lunatipula) zarnigor-group (Diptera, Tipulidae). Dopovidi Akademii Nauk Ukrayinskoyi RSR (B), 19704), 371-374 (in Ukrainian with Russian and English summaries).

1971 Savchenko, E.N. (1971a) Contributions to the Limoniid-flies fauna (Diptera, Limoniidae) of the USSR, 1. Subfamily Eriopterinae, genus Arctoconopa Al. Vestnik Zoologii, 1971(1), 1217 (in Russian).

Savchenko, E.N. (1970c) On a stenopterous form of Dicranomyia complicata de Meijere (Diptera, Limoniidae). Dopovidi Akademii Nauk Ukrayinskoyi RSR (B), 1970 (5), 466-469 (in Ukrainian with Russian and English summaries).

Savchenko, E.N. (1970d) A new subgenus and species of limoniids from the genus Dicranoptycha O.-S. (Diptera, Limoniidae). Dopovidi Akademii Nauk Ukrayinskoyi RSR (B), 1970(6), 563-566 (in Ukrainian with Russian and English summaries).

Savchenko, E.N. (1971b) A new brachypterous species of crane-fly of the genus Dactylolabis O.-S. (Diptera, Limoniidae) from the high mountain zone of Mongolia. Entomologicheskoe Obozrenie, 50 (1), 163-166 (in Russian) (English translation: Entomological Review, 50(1), 94-96 (1971)).

Savchenko, E.N. (1971c) New palaearctic species of Limoniid-flies (Diptera, Limoniidae), 1. The genus Prionolabis O.-S. Dopovidi Akademii Nauk Ukrayinskoyi RSR (B), 1971(8), 754756 (in Ukrainian with Russian and English summaries).

Savchenko, E.N. (1971d) New palaearctic species of Limoniid-flies (Diptera, Limoniidae), 2. The genus Antocha O.-S. Dopovidi Akademii Nauk Ukrayinskoyi RSR (B), 1971(9), 855-857 (in Ukrainian with Russian and English summaries).

Savchenko, E.N. (1971e) New palaearctic species of Limoniid-flies (Diptera, Limoniidae), 3. The genus Gonempeda Al. Dopovidi Akademii Nauk Ukrayinskoyi RSR (B), 1971(10), 949951 (in Ukrainian with Russian and English summaries). Savchenko, E.N. (1971f) Notes on palaearctic crane-flies (Diptera, Tipulidae). Zbirnyk Prats 
Zoolohichnoho Muzeyu, Kiev, 34, 53-58 (in Ukrainian with English summary). Savchenko, E.N. (1971g) Supplements to the crane-flies fauna (Diptera, Tipulidae) from the Magadan region. Zbirnyk Prats Zoolohichnoho Muzeyu, Kiev, 34, 58-59 (in Ukrainian with English summary).

Savchenko, E.N. (1971h) Afrolimnophila Al, a subgenus of Limoniid-flies (Diptera, Limoniidae) new for the palaearctic fauna. Vestnik Zoologii, 1971(6), 10-16 (in Russian with English summary).

Savchenko, E.N. (1971i) A new species of Austrolimnophila Al. (Diptera, Limoniidae). Doklady Akademii Nauk Tadzhikskoy SSR, 4(12), 58-60 (in Russian).

1972 Savchenko, E.N. (1972a) New palaearctic species of Limoniid-flies (Diptera, Limoniidae), 4. The genus Dicranota Ztt. Dopovidi Akademii Nauk Ukrayinskoyi RSR (B), 1972(2), 182-184 (in Ukrainian with English summary).

Savchenko, E.N. (1972b) The palaearctic Limoniid-flies (Diptera, Limoniidae) of the subgenus Cladolipes Lw. Dopovidi Akademii Nauk Ukrayinskoyi RSR (B), 1972(5), 466-470 (in Ukrainian with English summary).

Savchenko, E.N. (1972c) New palaearctic species of Limoniid-flies (Diptera, Limoniidae), 5. The genus Cheilotrichia Rossi. Dopovidi Akademii Nauk Ukrayinskoyi RSR (B), 1972(6), 560-562 (in Ukrainian with English summary).

Savchenko, E.N. (1972d) The Limoniid-flies (Diptera, Limoniidae) collected by the Soviet Mongolian expedition 1967-1969. Insects of Mongolia, 1,Izdatelstvo "Nauka", Leningrad, pp. 721-738 (in Russian).

Savchenko, E.N. (1972e) Additions to the fauna of crane-flies (Diptera, Tipulidae) of Mongolia. Insects of Mongolia, 1, Izdatelstvo "Nauka", Leningrad, pp. 739-740 (in Russian). Savchenko, E.N. (1972f) Contributions to the fauna of Limoniid-flies (Diptera, Limoniidae) of the USSR. The subfamily Eriopterinae, genus Mesocyphona O.-S. Vestnik Zoologii, 1972(5), 26-33 (in Russian).

Savchenko, E.N. (1972g) New palaearctic species of the genus Erioptera (Diptera, Limoniidae). Zoologicheskiy Zhurnal, 51(5), 671-681 (in Russian).

Savchenko, E.N. (1972h) New subgenera and species of the genus Gonomyia (Diptera, Limoniidae). Zoologicheskiy Zhurnal, 51(9), 1329-1341 (in Russian).

Savchenko, E.N. \& Sagdiyeva, P.D. (1972) A new Far-Eastern species of crane-fly from the Tipula (Lunatipula) fascipennis Mg. group (Diptera, Tipulidae). Dopovidi Akademii Nauk Ukrayinskoyi RSR (B), 1972(1), 83-86 (in Ukrainian with English summary).

Savchenko, E.N., Violovich, N.A. \& Narchuk, E.P. (1972) Review of the crane-flies (Diptera, Tipulidae) of the Altai mountains. Entomologicheskoe Obozrenie, 51(1), $74-95$ (in Russian) (English translation: Entomological Review, 51(1), 45-57 (1972)).

1973 Mannheims, B. \& Savchenko, E.N. (1973a) Ergebnisse der zoologischen Forschungen von Dr. Z. Kaszab in der Mongolei, 303. Tipulidae (Diptera), II. Folia Entomologica Hungarica, (N.S.), Supplement 26, 157-186.

Mannheims, B. \& Savchenko, E.N. (1973b) Ergebnisse der zoologischen Forschungen von Dr. Z. Kaszab in der Mongolei, 304. Limoniidae (Diptera). Annales Historico-Naturales Musei Nationalis Hungarici, 65, 257-269.

Savchenko, E.N. (1973a) Crane-flies (Fam. Tipulidae), Subfam. Tipulinae (end) and Flabelliferinae. Fauna USSR, New Series N 105, Nasekomye dvukrylye [Diptera], 2(5), 282 pp. Izdatelstvo "Nauka”, Leningradskoe otdelenie. Leningrad [Publishing house of Academie of Sciences of the USSR]. (in Russian).

Savchenko, E.N. (1973b) A new Limoniid-fly (Diptera, Limoniidae) from the Paradelphomyia (Oxyrhiza) nipponensis Al. group. Dopovidi Akademii Nauk Ukrayinskoyi RSR (B), 1973(3, 280-282 (in Ukrainian with English summary). 
Savchenko, E.N. (1973c) Distribution and new sibling species of the Limoniid-fly Phylidorea (s. str.) ferruginea (Mg.) (Diptera, Limoniidae). Dopovidi Akademii Nauk Ukrayinskoyi RSR (B), 1973(4), 371-373 (in Ukrainian with English summary).

Savchenko, E.N. (1973d) New and little-known palaearctic species of Limoniid-flies (Diptera, Limoniidae), 1. The genera Ormosia Rond. and Scleroprocta Edw. Entomologicheskoe Obozrenie, 52 (2), 440-462 (in Russian) (English translation: Entomological Review, 52(2), 303-320 (1973)).

Savchenko, E.N. (1973e) New species of Limoniid-flies (Diptera, Limoniidae) from the South of the USSR. Dopovidi Akademii Nauk Ukrayinskoyi RSR (B), 1973(6), 568-571 (in Ukrainian with English summary).

Savchenko, E.N. (1973f) Additions to the crane-flies (Diptera, Tipulidae) fauna of the Transcaucasus. Zbirnyk Prats Zoolohichnoho Muzeyu, Kiev, 35, 29-38 (in Ukrainian).

Savchenko, E.N. (1973g) Two new species of Limoniid-flies (Diptera, Limoniidae) from the Ukrainian Carpathians. Zbirnyk Prats Zoolohichnoho Muzeyu, Kiev, 35, 38-42 (in Ukrainian).

1974 Savchenko, E.N. (1974a) The palaearctic species of Limoniid-flies (Diptera, Limoniidae) from the Ilisia maculata (Meigen, 1804) group. Zoologicheskiy Zhurnal, 53(3), 473-477 (in Russian with English summary).

Savchenko, E.N. (1974b) Two new species of Limoniid-flies (Diptera, Limoniidae) from the mountains of Middle Asia. Dopovidi Akademii Nauk Ukrayinskoyi RSR (B), 1974(5), 470 473 (in Ukrainian with English summary).

Savchenko, E.N. (1974c) New palaearctic species of Limoniid-flies (Diptera, Limoniidae), 6. The genera Dicranomyia Steph. and Rhipidia Mg. Vestnik Zoologii, 1974(4), 65-72 (in Russian).

Savchenko, E.N. (1974d) Dipteren aus der Mongolischen Volksrepublik (Diptera, Insekta). Ergebnisse der Mongolisch-Deutschen Biologischen Expeditionen seit 1962. Nr. 65. Mitteilungen aus dem Zoologischen Museum in Berlin, 50(2), 223-232. [Tipulidae 223-224, Limoniidae 224]

Savchenko, E.N. (1974e) The palaearctic species of Limoniid-flies (Diptera, Limoniidae) of the Cheilotrichia (s. str.) exigua Lack. group. Dopovidi Akademii Nauk Ukrayinskoyi RSR (B), 1974(10), 942-946 (in Ukrainian with English summary).

Savchenko, E.N. (1974f) New palaearctic species of Limoniid-flies (Diptera, Limoniidae), 7. The genera Dicranoptycha O.-S., Orimarga O.-S. and Thaumastoptera Mik. Vestnik Zoologii, 1974(6), 31-36 (in Russian).

Savchenko, E.N. \& Parkhomenko, S.I. (1974) On the Limoniid-flies fauna (Diptera, Limoniidae) of the Turkmen SSR. Dopovidi Akademii Nauk Ukrayinskoyi RSR (B), 1974(11), 1048-1051 (in Ukrainian with English summary).

(1)

Savchenko, E.N. (1975) On taxonomy and geographical distribution of the Limoniid-fly Dicranoptycha (Ulugbekia) mirabilis Sav. (Diptera, Limoniidae). Dopovidi Akademii Nauk Ukrayinskoyi RSR (B), 1975(11), 1040-1043 (in Ukrainian), 1042-1045 (in Russian, Doklady edition) (with English summary).

Savchenko, E.N. \& Tomov, V. (1975) A new species of Ormosia (Diptera, Limoniidae) from Bulgaria. Dopovidi Akademii Nauk Ukrayinskoyi RSR RSR (B), 1975(10), 948-950 (in

Ukrainian), 950-952 (in Russian, Doklady edition) (with English summary).

North-West Caucasus. Zoologicheskiy Zhurnal, 55(2), 304-306 (in Russian with English summary). 
Russian with English summary).

Savchenko, E.N. (1976c) Molophilus (s. str.) ermolenkoi Sav., a new species of the Limoniidflies (Diptera, Limoniidae) from the Ukrainian Carpathians. Dopovidi Akademii Nauk Ukrayinskoyi RSR (B), 1976(4), 370-373 (in Ukrainian), 373-377 (in Russian, Doklady edition) (with English summary).

Savchenko, E.N. (1976d) New or little-known species of palaearctic Limoniidae (Diptera), 2. Subfam. Eriopterinae, genus Molophilus Curt. Entomologicheskoe Obozrenie, 55(2), 438451 (in Russian) (English translation: Entomological Review, 55(2), 126-136 (1976)).

Savchenko, E.N. (1976e) On the fauna of Limoniid-flies (Diptera, Limoniidae) of the USSR, 1. Genus Elephantomyia O.-S. Dopovidi Akademii Nauk Ukrayinskoyi RSR (B), 1976(5), 466-470 (in Ukrainian), 469-472 (in Russian, Doklady edition) (with English summary).

Savchenko, E.N. (1976f) On finding Limoniid-flies (Diptera, Limoniidae) of the subgenus Palaeogonomyia Meunier of the genus Rhabdomastix Skuse in the fauna of the USSR. Dopovidi Akademii Nauk Ukrayinskoyi RSR (B), 1976(6), 561-564 (in Ukrainian), 561-565 (in Russian, Doklady edition) (with English summary).

Savchenko, E.N. \& Krivolutskaya, G.O. (1976) Limoniidae of the South Kuril Islands and south Sakhalin. Akademiy Nauk Ukrainskoy SSR, Institut Zoologii, Izdatelstvo "Naukova Dumka", Kiev, 160 pp. (in Russian).

Starý, J. \& Savchenko, E.N. (1976) Eine neue Limonia-Art aus der Krim und vom Kaukasus (Diptera, Limoniidae). Acta Entomologica Bohemoslovaca, 73(3), 183-186.

Savchenko, E.N. (1977a) On the hydro- and helobiont fauna of Limoniid-flies (Diptera, Limoniidae) of the nature reserve Kedrovaja Pad and some other districts of South Primorye. Trudy Biologo-Pochvennogo Instituta DVNC AN SSSR, Vladivostok, (N.S.) 45(148), 88-108 (in Russian).

Savchenko, E.N. (1977b) The Limoniid-flies fauna (Diptera, Limoniidae) of Chukotka and neighbouring districts of Eastern Siberia. Trudy Biologo-Pochvennogo Instituta DVNC AN SSSR, Vladivostok, (N.S.) 46(149), 75-92 and [Ispravleniya] Erratum (in Russian).

Savchenko, E.N. (1977c) A new genus and species of Limoniid-flies (Diptera, Limoniidae) from the Far East of the USSR. Dopovidi Akademii Nauk Ukrayinskoyi RSR (B), 1977(6), 564-568 (in Ukrainian), 566-570 (in Russian, Doklady edition) (with English summary).

117

Savchenko, E.N. (1978a) The fauna of Limoniid-flies (Diptera, Limoniidae) of the North Kuriles. In: Systematics and biology of the freshwater organisms of North-East Asia. Trudy Biologo-Pochvennogo Instituta DVNC AN SSSR, Vladivostok, (N.S.) 49(152), 63-68 (in Russian).

Savchenko, E.N. (1978b) The palaearctic species of Limoniid-flies (Diptera, Limoniidae) of the subgenus Archilimnophila Al. of the genus Austrolimnophila Al. Dopovidi Akademii Nauk Ukrayinskoyi RSR (B), 1978(4), 376-378 (in Ukrainian), 378-381 (in Russian, Doklady edition) (with English summary).

Savchenko, E.N. (1978c) New or little-known species of palaearctic Limoniidae (Diptera), 3. Subfam. Eriopterinae, genus Molophilus Curt. Entomologicheskoe Obozrenie, 57(2), 399415 (in Russian) (English translation: Entomological Review, 57(2), 280-292 (1978)). Savchenko, E.N. (1978d) Species of the crane-fly genus Ctenophora Mg. (Diptera, Tipulidae) new for the fauna of the USSR. Dopovidi Akademii Nauk Ukrayinskoyi RSR (B), 1978(6), 563-566 (in Ukrainian), 567-570 (in Russian, Doklady edition) (with English summary). Savchenko, E.N. (1978e) A new species of Arctotipula (Diptera, Tipulidae) from the East of the USSR. Dopovidi Akademii Nauk Ukrayinskoyi RSR (B), 1978(7), 652-655 (in Ukrainian), 656-660 (in Russian, Doklady edition) (with English summary).

Savchenko, E.N. (1978f) New and little-known species and subspecies of Limoniid-flies (Diptera, Limoniidae) from the USSR, 1. Genus Pedicia, subgenus Crunobia. Zoologicheskiy 
Zhurnal, 57(5), 700-713 (in Russian with English summary).

Savchenko, E.N. (1978g) New or little-known Limoniid-flies (Diptera, Limoniidae) from the USSR, 2. The genus Dactylolabis, nominative subgenus. Zoologicheskiy Zhurnal, 57(8), 1175-1188 (in Russian with English summary).

Savchenko, E.N. (1978h) Additions to the Limoniid-flies fauna (Diptera, Limoniidae) of the North Tien Shan. Vestnik Zoologii, 1978(5), 40-49 (in Russian).

Savchenko, E.N. (1978i) Change of name. Vestnik Zoologii, 1978(5), 49 (in Russian).

Savchenko, E.N. \& Parkhomenko, S.I. (1978) On the Limoniid-flies fauna (Diptera, Limoniidae) of the Northern Tien Shan. Vestnik Zoologii, 1978(1), 29-34 (in Russian).

Savchenko, E.N. \& Theischinger, G. (1978) Die Arten der Tipula (Lunatipula) recticornisGruppe. Bulletin Zoologisch Museum, Universiteit van Amsterdam, 6 (16), 117-128.

Savchenko, E.N. (1979a) New species of Limoniid-flies (Diptera, Limoniidae) of the genus Limonia Mg. from the USSR. Entomologicheskoe Obozrenie, 58(1), 153-160 (in Russian) (English translation: Entomological Review, 58(1), 87-92 (1979)).

Savchenko, E.N. (1979b) A new subgenus and new species of Limoniid-flies (Diptera, Limoniidae) of the genus Neolimnomyia Séguy. Dopovidi Akademii Nauk Ukrayinskoyi RSR (B), 1979(6), 482-485 (in Ukrainian), 484-487 (in Russian, Doklady edition) (with English summary).

Savchenko, E.N. (1979c) Limoniid-flies (Diptera, Limoniidae) from the genus Molophilus Curt. new for the fauna of the USSR. Dopovidi Akademii Nauk Ukrayinskoyi RSR (B), 1979(8), 680-684 (in Ukrainian), 683-687 (in Russian, Doklady edition) (with English summary).

Savchenko, E.N. (1979d) Supplements and corrections to the Limoniid-flies fauna (Diptera, Limoniidae) of South Kuriles and South Sakhalin. Vestnik Zoologii, 1979(1), 23-29 (in Russian).

Savchenko, E.N. (1979e) A new species of the genus Dicranota (Diptera, Limoniidae) from the South-West Transcaucasus. Zoologicheskiy Zhurnal, 58 (9), 1422-1425 (in Russian).

Savchenko, E.N. (1979f) Phylogenie und Systematik der Tipulidae. Translated and revised by Br. Theowald and G. Theischinger. Tijdschrift voor Entomologie, 122 (5), 91-126.

Savchenko, E.N. (1979g) Two new species of Caucasian Limoniid-flies (Diptera, Limoniidae) from the Eriopterinae subfamily. Dopovidi Akademii Nauk Ukrayinskoyi RSR (B), 1979(9), 769-773 (in Ukrainian), 771-775 (in Russian, Doklady edition) (with English summary).

Savchenko, E.N. (1979h) Species of the crane-fly genus Dolichopeza Curt. (Diptera, Tipulidae) from the Far-East. In: Ler, P.A. (ed.), Land arthropods of the Far East. Far Eastern Scientific Center of Academy of Sciences of the USSR, Institute of Biology and Soil Sciences, Vladivostok, pp. 108-116 (in Russian).

Savchenko, E.N. (1979i) The Limoniid-flies fauna (Diptera, Limoniidae) of the Chukot Peninsula. Dopovidi Akademii Nauk Ukrayinskoyi RSR (B), 1979(12), 1053-1056 (in Ukrainian), 1054-1057 (in Russian, Doklady edition) (with English summary).

Savchenko, E.N. (1980a) Two genera new for the palaearctic fauna of Limoniid-flies (Diptera, Limoniidae) and two new species of these genera. Vestnik Zoologii, 1980(1), 17-25 (in Russian).

Savchenko, E.N. (1980b) New and little-known species of Limoniidae (Diptera) from the Zailijskij Alatau. Entomologicheskoe Obozrenie, 59 (2), 391-403 (in Russian) (English translation: Entomological Review, 59 (2), 134-145 (1980)).

Savchenko, E.N. (1980c) Two new species of Gonomyia (Diptera, Limoniidae) from Sovjet Middle Asia. Vestnik Zoologii, 1980(3), 36-41 (in Russian).

Savchenko, E.N. (1980d) On the crane-fly fauna (Diptera) of the USSR. Subfamily 
Dolichopezinae, Genus Oropeza Needh. In: Taxonomy of the Insects of the Far East. Far Eastern Scientific Center of Academy of Sciences of the USSR, Institute of Biology and Soil Sciences, Vladivostok, pp. 109-114 (in Russian).

Savchenko, E.N. (1980e) On the diagnosis and distribution of the Limoniid-fly Arctoconopa forcipata (Lundstrom) (Diptera, Limoniidae). In: Freshwater Fauna of the Far East. Far Eastern Scientific Center of Academy of Sciences of the USSR, Institute of Biology and Soil Sciences, Vladivostok, pp. 110-112 (in Russian).

Savchenko, E.N. \& Parkhomenko, S.I. (1980) Supplementary data on the Limoniid-flies (Diptera, Limoniidae) fauna of Chukotka. In: Freshwater Fauna of the Far East. Far Eastern Scientific Center of Academy of Sciences of the USSR, Institute of Biology and Soil Sciences, Vladivostok, pp. 95-109 (in Russian).

Savchenko, E.N. (1981a) Three new species of Limoniid-flies (Diptera, Limoniidae) from the USSR. Vestnik Zoologii, 1981(1), 22-29 (in Russian).

Savchenko, E.N. (1981b) On the systematic position of the Limoniid-fly Chionea crassipes Boh. (Diptera, Limoniidae) whithin genus. Vestnik Zoologii, 1981(2), 74-76 (in Russian).

Savchenko, E.N. (1981c) A species of crane-flies (Diptera, Tipulidae) new to the fauna of the USSR, Tipula (Platytipula) demarcata (Brunetti, 1912). Vestnik Zoologii, 1981(3), 10 (in Russian).

Limoniidae)? Vestnik Zoologii, 1982(1), 74 (in Russian). Limoniidae). Vestnik Zoologii, 1982(2), 62 (in Russian).

Savchenko, E.N. (1981d) Tipula (Savtshenkia) koreana Alexander, 1935, a species of craneflies new to the fauna of the USSR. Vestnik Zoologii, 1981(3), 10 (in Russian).

Savchenko, E.N. (1981e) A species of genus Nephrotoma new to the fauna of the USSR, N. daisensis Alexander, 1935. Vestnik Zoologii, 1981(3), 77 (in Russian).

Savchenko, E.N. (1981f) Antocha (Proantocha) serricauda Al., a species of Limoniid-flies new to the fauna of the USSR. Vestnik Zoologii, 1981(4), 50 (in Russian).

Savchenko, E.N. (1981g) Species of Limoniid-flies (Diptera, Limoniidae) new to the fauna of Turkmenistan. Vestnik Zoologii, 1981(5), 91 (in Russian).

Savchenko, E.N. (1981h) Two new Caucasian species of the genus Erioptera (Diptera, Limoniidae). Zoologicheskiy Zhurnal, 9(10), 1585-1588 (in Russian with English summary). Savchenko, E.N. (1981i) Idiocera (s. str.) laterospina Al., a species of Limoniid-flies (Diptera, Limoniidae) new to the fauna of the USSR. Vestnik Zoologii, 1981(6), 66 (in Russian).

Savchenko, E.N. (1981j) Fam. Tipulidae—crane-flies or karamory. In: Insects and mites pests of agricultural crops. Vol. IV. Hymenoptera and Diptera. Zoological Institute of Academy of Sciences of the USSR, Leningrad, "Nauka", Leningrad division, pp. 57-63 (in Russian).

Savchenko, E.N. (1981k) Fam. Limoniidae - limoniids flies. In: Insects and mites - pests of agricultural crops. Vol. IV. Hymenoptera and Diptera. Zoological Institute of Academy of Sciences of the USSR, Leningrad, "Nauka", Leningrad division, pp. 64 (in Russian).

Savchenko, E.N. (1982c) A new Psiloconopa (Diptera, Limoniidae) from West Siberia. Vestnik Zoologii, 1982(2), 70-72 (in Russian).

Savchenko, E.N. (1982d) On the distribution of the Limoniid-fly Atypophthalmus inustus (Mg.) (Diptera, Limoniidae). Vestnik Zoologii, 1982(4), 62 (in Russian).

Savchenko, E.N. (1982e) Change of name in the family of Limoniid-flies (Diptera, Limoniidae). Vestnik Zoologii, 1982(4), 81 (in Russian).

Savchenko, E.N. (1982f) On the systematics and synonymy of the Limoniid-flies (Diptera, Limoniidae) Limnophila posthabita and L. tetrasticta. Vestnik Zoologii, 1982(5), 80-83 (in 
Russian).

161

Savchenko, E.N. (1982g) Komari-limonijidi [Limoniid-flies], (subfamily Eriopterinae). In: Fauna Ukrajiny, Dovgovusi dvokrili, Vol. 14(3) I.I. Schmalhausen Institute of Zoology of Academy of Sciences of Ukraine, "Naukova Dumka", Kiev, 335 pp. (in Ukrainian).

Savchenko, E.N. \& Rybin, S.N. (1982) On the Limoniidae fauna (Diptera) of the Alai range. Entomologicheskoe Obozrenie, 61(2), 392-403 (in Russian) (English translation: Entomological Review, 61(2), 142-154 (1982)).

Savchenko, E.N. (1983a) Crane-flies (Fam. Tipulidae), Introduction, and beginning of systematic part. Subfam. Dolichopezinae, subfam. Tipulinae (start). Fauna USSR, New Series N 127, Nasekomye dvukrylye [Diptera], 2(1-2), 586 pp. Leningrad, "Nauka" Leningradskoe otdelenie. [Publishing house of Academie of Sciences of the USSR]. (in Russian).

Savchenko, E.N. (1983b) Three new species of Limoniid-flies (Diptera, Limoniidae) of the fauna of the USSR. Vestnik Zoologii, 1983(1), 13-20 (in Russian).

Savchenko, E.N. (1983c) A new subspecies of Molophilus (Diptera, Limoniidae) from Armenia. Vestnik Zoologii, 1983(1), 52 (in Russian).

Savchenko, E.N. (1983d) New data on the distribution of Limoniid-flies (Diptera, Limoniidae) in the USSR. Vestnik Zoologii, 1983(1), 84 (in Russian).

Savchenko, E.N. (1983e) Gonomyia (s. str.) papposa sp.n. from the Armenian SSR (Diptera, Limoniidae). Zoologicheskiy Zhurnal, 62(4), 643-645 (in Russian with English summary).

Savchenko, E.N. (1983f) The Limoniid-flies (Diptera, Limoniidae) of the Ilisia (Parilisia) fausta (Al.) group in the Sovjet Middle Asia fauna. Vestnik Zoologii, 1983(4), 3-8 (in Russian).

Savchenko, E.N. (1983g) A Molophilus (Diptera, Limoniidae) new for the fauna of the Ukraine. Vestnik Zoologii, 1983(5), 31 (in Russian).

Savchenko, E.N. (1983h) Limoniidae of South Primorye. Akademiy Nauk Ukrainskoy SSR, I.I. Schmalhausen Institute of Zoology of Academy of Sciences of Ukraine, "Naukova Dumka", Kiev, 156 pp, (in Russian).

Savchenko, E.N. \& Parkhomenko, S.I. (1983) A new polymorphic species of Paradicranota (Diptera, Limoniidae) from the North-West Caucasus. Zoologicheskiy Zhurnal, 62 (9), $1353-$ 1358 (in Russian with English summary).

Savchenko, E.N. (1984a) A new subspecies of Ilisia (Diptera, Limoniidae) from Crimea. Vestnik Zoologii, 1984(1), 88 (in Russian).

Savchenko, E.N. (1984b) A new subgenus of Limoniid-flies (Diptera, Limoniidae) for the fauna of the USSR. Vestnik Zoologii, 1984(2), 7 (in Russian).

Savchenko, E.N. (1984c) On the tipuloid fauna (Diptera, Tipulidae) of the Bering Island. Vestnik Zoologii, 1984(2), 7 (in Russian).

Savchenko, E.N. (1984d) The palaearctic species of the Limoniid-flies (Diptera, Limoniidae) from the Dicranomyia (s. str.) goritiensis (Mik) group. In: Taxonomy and Zoogeography of Insects, Akademiy Nauk Ukrainskoy SSR, I.I. Schmalhausen Institute of Zoology of Academy of Sciences of Ukraine, "Naukova Dumka”, Kiev, pp. 95-102 (in Russian).

Savchenko, E.N. (1984e) A species of Limoniid-flies (Diptera, Limoniidae) new for the fauna of the Ukraine. Vestnik Zoologii, 1984(5), 70 (in Russian).

Savchenko, E.N. \& Plyushch, G.I. (1984) On the fauna of Limoniidae (Diptera) of the Gissar mountains, 1. Entomologicheskoe Obozrenie, 63(1), 166-185 (in Russian) (English translation: Entomological Review, 63(2), 1-21 (1984)).

Savchenko, E.N. (1985a) On the distribution of the Limoniid-fly (Diptera, Limoniidae) Austrolimnophila (Archilimnophila) harperi Al. Vestnik Zoologii, 1985(3), 40 (in Russian).

Savchenko, E.N. (1985b) Komary-limoniidy [Limoniid-flies]. Subfamily Limoniinae. In: Fauna Ukrainy, Dlinnousye dvukrilie, [Long-horned flies], 14(4), I.I. Schmalhausen Institute 
of Zoology of Academy of Sciences of Ukraine, "Naukova Dumka", Kiev, 180 pp. (in Russian).

Savchenko, E.N. \& Plyushch, G.I. (1985) On the limoniid-flies (Diptera, Limoniidae) fauna of the Gissar mountains, 2. Entomologicheskoe Obozrenie, 64(3), 616-625 (in Russian).

Zoologii, 1986(1), 29-35 (in Russian).

Savchenko, E.N. (1986b) On the distribution of the Limoniid-fly (Diptera, Limoniidae) Erioptera (s. str.) cornuta Sav. Vestnik Zoologii, 1986(2), 75 (in Russian).

Savchenko, E.N. (1986c) Komary-limoniidy [Limoniid-flies]. (General description, subfamilies Pediciinae and Hexatominae). In: Fauna Ukrainy, Dlinnousye dvukrilie, [Long-horned flies], 14(2), I.I. Schmalhausen Institute of Zoology of Academy of Sciences of Ukraine, "Naukova Dumka", Kiev, 380 pp. (in Russian).

Savchenko, E.N. (1986d) Palaearctic Limoniid flies (Diptera, Limoniidae) of the nigronotata group of the genus Phylidorea. Vestnik Zoologii, 1986(5), 20-26 (in Russian).

Savchenko, E.N. (1986e) Two species of Limoniid-flies (Diptera, Limoniidae) new for the fauna of the USSR. Vestnik Zoologii, 1986(5), 87 (in Russian).

1987 Savchenko, E.N. (1987a) A new species of the genus Gonomyia (Diptera, Limoniidae). Zoologicheskiy Zhurnal, 66(2), 304-307 (in Russian with English summary).

Savchenko, E.N. (1987b) A species of Limoniid-flies (Diptera, Limoniidae) new for the fauna of the USSR, Limonia messaurea Mendl. Vestnik Zoologii, 1987(6), 78 (in Russian).

Savchenko, E.N. (1987c) Idiopyga Savtshenko nom. nov. (Diptera, Limoniidae). Vestnik Zoologii, 1987(6), 81 (in Russian).

Savchenko, E.N. \& Kandybina, M.N. (1987a) Limoniidae. In: Kandybina, M.N., Lantsov, V.I. and Savchenko, E.N. (eds), A catalog of the type-specimens in the collection of the Zoological Institute, Academy of Sciences of the USSR. Insecta, Diptera, 3, Families Tanyderidae, Trichoceridae, Limoniidae, Tipulidae. Zoological Institute of Academy of sciences of the USSR, Leningrad, "Nauka", pp. 8-20 (in Russian).

Savchenko, E.N. \& Kandybina, M.N. (1987b) Tipulidae. In: Kandybina, M.N., Lantsov, V.I. and Savchenko, E.N. (eds), A catalog of the type-specimens in the collection of the Zoological Institute, Academy of Sciences of the USSR. Insecta, Diptera, 3, Families Tanyderidae, Trichoceridae, Limoniidae, Tipulidae. Zoological Institute of Academy of sciences of the USSR, Leningrad, "Nauka", pp. 21-61 (in Russian).

1988 Savchenko, E.N. (1988a) Limoniid flies (Diptera, Limoniidae) new for the fauna of Kirghizia. Vestnik Zoologii, 1988(1), 50 (in Russian).

Savchenko, E.N. (1988b) A new crane-fly species from the Eastern Yakutia (Diptera, Tipulidae). Vestnik Zoologii, 1988(3), 19-22 (in Russian).

Savchenko, E.N. (1989a) Family Cylindrotomidae. In: Bei-Beinko, G.Y. (ed.), Key to the insects of the European part of the USSR, 5, Diptera and Siphonaptera. Part 1. Smiths. Inst. Libr. and Nat. Res. Found., Washington DC, pp. 72-74. (English version of Savchenko, 1969e.)

Savchenko, E.N. (1989b) Family Tipulidae. In: Bei-Beinko, G.Y. (ed.), Key to the insects of the European part of the USSR, 5, Diptera and Siphonaptera. Part 1. Smiths. Inst. Libr. and Nat. Res. Found., Washington DC, pp. 75-118. (English version of Savchenko, 1969f.) Savchenko, E.N. (1989c) Family Limoniidae. In: Bei-Beinko, G.Y. (ed.), Key to the insects of the European part of the USSR, 5, Diptera and Siphonaptera. Part 1. Smiths. Inst. Libr. and Nat. Res. Found., Washington DC, pp. 119-163. (English version of Savchenko, 1969g.) Savchenko, E.N. (1989d) Komary-limoniidy fauny SSSR [Limoniidae fauna of the USSR]. Determination tables of superspecies taxa with catalogue survey of species. Akademiy Nauk Ukrainskoy SSR, I.I. Schmalhausen Institute of Zoology of Academy of 
Sciences of Ukraine, "Naukova Dumka”, Kiev, 380 pp. (in Russian).

1961991 Savchenko, E.N., Plyushch, I.G. \& Rybin, S.N. (1991) New data on the fauna of the Limoniidae (Diptera) of Kirghizia. Entomologicheskoe Obozrenie, 70(3), 686-693 (in Russian) (English translation: Entomological Review, 71(4), 62-69 (1992)).

1971992 Savchenko, E.N., Oosterbroek, P. \& Starý, J. (1992) Family Limoniidae. In: Catalogue of Palaearctic Diptera, 1, Trichoceridae - Nymphomyiidae. Hungarian Natural History Museum, Budapest, pp. 183-369. 